\title{
Electron and muon anomalous magnetic moments in the inverse seesaw extended NMSSM
}

\author{
Junjie Cao, ${ }^{*}$ Yangle He, ${ }^{\dagger}$ Jingwei Lian, ${ }^{\dagger}$ Di Zhang $\odot,{ }^{\S}$ and Pengxuan Zhu® ${ }^{\|}$ \\ Department of Physics, Henan Normal University, Xinxiang 453007, China
}

(Received 26 March 2021; accepted 9 August 2021; published 8 September 2021)

\begin{abstract}
The recently improved observation of the fine structure constant has led to a negative $2.4 \sigma$ anomaly of electron $g-2$. Combined with the long-existing positive $4.2 \sigma$ discrepancy of the muon anomalous magnetic moment, it is interesting and difficult to explain these two anomalies with a consistent model without introducing flavor violations. We show that they can be simultaneously explained in the inverse seesaw extended next-to-minimal supersymmetric standard model by the Higgsino-sneutrino contributions to $(g-2)_{e}$ and $(g-2)_{\mu}$. The spectrum features prefer light $\mu$, which can predict $m_{Z}$ naturally, and it is not difficult to obtain a $\tau$-type sneutrino dark matter candidate that is compatible with the observed dark matter relic density and the bounds from dark matter direct detection experiments. Due to the compressed spectra and the undetectable decay mode of selectrons, they can evade the current Large Hadron Collider constraints.
\end{abstract}

DOI: 10.1103/PhysRevD.104.055009

\section{INTRODUCTION}

Since Schwinger showed that $a_{\ell} \equiv\left(g_{\ell}-2\right) / 2=\frac{\alpha}{2 \pi}[1]$, the anomalous magnetic moments of charged leptons have survived rigorous tests of the quantum electrodynamics and the later Standard Model (SM) of particle physics for more than half a century. Recently, an improvement of the measurement of the fine structure constant $\alpha$, via the recoil frequency of cesium-133 atoms, has yielded the most accurate measurement [2]:

$$
\alpha^{-1}(\mathrm{Cs})=137.035999046(27) .
$$

As a result, there is a negative $2.4 \sigma$ discrepancy between the theoretical prediction $a_{e}^{\mathrm{SM}}[3]$ and the existing experimental measurement $a_{e}^{\exp }[4,5]$ of the electron anomalous magnetic moment,

$$
\Delta a_{e} \equiv a_{e}^{\exp }-a_{e}^{\mathrm{SM}}=(-87 \pm 36) \times 10^{-14} .
$$

Meanwhile, the long-standing discrepancy of the muon anomalous magnetic moment [6] between the SM

\footnotetext{
junjiec@alumni.itp.ac.cn

heyangle@htu.edu.cn

*ljwfly@ hotmail.com

\$dz481655@gmail.com

"zhupx99@icloud.com
}

Published by the American Physical Society under the terms of the Creative Commons Attribution 4.0 International license. Further distribution of this work must maintain attribution to the author(s) and the published article's title, journal citation, and DOI. Funded by SCOAP ${ }^{3}$. prediction $a_{\mu}^{\mathrm{SM}}[7-26]$ and the combined results $a_{\mu}^{\exp }$ of Fermilab Muon g-2 experiment [27] and the E821 experiment of Brookhaven National Laboratory $[28,29]$ is

$$
\Delta a_{\mu} \equiv a_{\mu}^{\exp }-a_{\mu}^{\mathrm{SM}}=(251 \pm 59) \times 10^{-11},
$$

corresponding to a $4.2 \sigma$ discrepancy.

There is insufficient evidence to show that these two anomalies are indeed signs of new physics (NP). The discovery level confirmation of $\Delta a_{\mu}$, for example, requires efforts from the currently running E989 experiment at the Fermilab and the future J-PARC experiment and also progress in reducing the theoretical uncertainty. Providing a common explanation to these two anomalies in an NP model is very challenging. In general, in a complete renormalizable model, $a_{\ell}$ can only be a quantum loop effect, because it comes from a dimension-5 operator. In a generic NP model without flavor violation, the new contribution to the anomalous magnetic moment $a_{\ell}^{\mathrm{NP}}$ is proportional to the mass square of the lepton times an NP factor $R_{\ell}^{\mathrm{NP}}$. Taking the central values of the two anomalies in Eqs. (1.2) and (1.3), one can easily find that there needs to be a difference of about -15 between $R_{e}^{\mathrm{NP}}$ and $R_{\mu}^{\mathrm{NP}}$,

$$
\frac{R_{e}^{\mathrm{NP}}}{R_{\mu}^{\mathrm{NP}}}=\frac{m_{\mu}^{2}}{m_{e}^{2}} \frac{\Delta a_{e}}{\Delta a_{\mu}} \sim-15,
$$

which is difficult to achieve from a common physical origin.

At present, there have already been several discussions offering combined explanations of the experimental results 
for electron and muon anomalous magnetic moments [30-64]. Among these discussions, the supersymmetry (SUSY) framework includes a chiral enhancement factor $\tan \beta$, which has shown promising results [48]. Reference [30] argued that the combined explanation in the SUSY framework needs relatively large nonuniversal trilinear $A$ terms and also requires a flavor violation (for a more detailed discussion, see Refs. $[49,65])$. Due to the constraint from the lepton flavor violating process, Ref. [47] examined the minimal flavor violation within the minimal supersymmetric standard model (MSSM) and found its compatibility with the Higgs mediation scenario. However, since the value of parameter $\mu$ needs to be at $\mathcal{O}(100 \mathrm{TeV})$, the parameter space of the explanation in Ref. [47] is unattractive.

More recently, Ref. [44] argued that in the MSSM without any flavor violation, a combined explanation can be achieved by setting the conditions that $\operatorname{sgn}\left(M_{1} \mu\right)<0$ and $\operatorname{sgn}\left(M_{2} \mu\right)>0$. The corresponding result features very light selectrons and winolike charginos, which avoid the Large Hadron Collider (LHC) constraint due to their degenerate spectra. The solution of Ref. [44] is impressive, but it also has two unsatisfactory characteristics. One is that the solution prefers heavy Higgsinos with masses $\mu \sim \mathcal{O}(1 \mathrm{TeV})$. This leads to a relatively fine-tuned electroweak sector. In general, $\mu$ should be close to the $Z$ boson mass $m_{Z}$ to avoid large cancellation when predicting the observed value of $m_{Z}=91.2 \mathrm{GeV}$ [66-70]. $\mu \sim 1 \mathrm{TeV}$ often induces tuning on the order of $1 / 10000$ to predict $m_{Z}$. The other is that winolike particles are too light due to the current restrictions of the LHC direct SUSY searches. The wino exclusion planes reported by ATLAS and CMS within the simplified model framework are appropriate for the scenario of Ref. [44]. According to Fig. 8 in the CMS report [71], for example, the benchmark points in Ref. [44] with $M_{2} \sim 200 \mathrm{GeV}$ are on the verge of being excluded by the multilepton plus $E_{\mathrm{T}}^{\text {miss }}$ signal via the electroweakino channel $p p \rightarrow \tilde{\chi}_{1}^{ \pm} \tilde{\chi}_{2}^{0}$.

In our previous work [72], we investigated the observation that in the inverse-seesaw mechanism extended next-to-minimal supersymmetric standard model (ISSNMSSM), due to the $\mathcal{O}(0.1)$ level Yukawa coupling $Y_{\nu}$ of the Higgs field to the right-handed neutrino, the Higgsino-sneutrino (HS) loop can be a new source of $a_{\mu}$ to explain $\Delta a_{\mu}$. Unlike the MSSM, the newly introduced HS contribution $a_{\ell}^{\mathrm{HS}}$ in the ISS-NMSSM prefers a light $\mu$. The sign of $a_{\ell}^{\mathrm{HS}}$ is determined by the mass mixing effect of sneutrino fields $\tilde{\nu}_{L}^{\ell}, \tilde{\nu}_{R}^{\ell}$, and $\tilde{\nu}_{x}^{\ell}$ for a given flavor $\ell$, not by the mixing of charginos or neutralinos. In the ISS-NMSSM explanation, the masses of winolike particles can be much heavier than the current LHC bounds. One can also assume one generation of sneutrinos to be the lightest supersymmetric particles (LSPs), which act as a dark matter (DM) candidate coannihilating with Higgsinos to achieve the observed relic density. Due to the singlet nature, the
DM-nucleus scattering cross section is naturally suppressed below the current experimental detection limits [72-74]. In this case, the neutral Higgsinos are the next-to-lightest supersymmetric particles (NLSPs) that decay into the invisible final states of the collider $\left(\tilde{H}^{0} \rightarrow \nu \tilde{\nu}\right)$. The charged Higgsino decays into a soft charged lepton and DM $\left(\tilde{H}^{ \pm} \rightarrow \ell^{ \pm} \tilde{\nu}\right)$. Due to the lower production rate than that of winos and the degenerate mass spectrum, the current LHC data still allow a low Higgsino mass of around $100 \mathrm{GeV}$. Thus, compared with the MSSM framework, the ISS-NMSSM is more natural for providing common explanations for $\Delta a_{e}$ and $\Delta a_{\mu}$.

In this work, we investigate this issue by applying the ISS-NMSSM to explain $\Delta a_{e}$ and $\Delta a_{\mu}$. The remainder of this paper is organized as follows. First, we briefly introduce the ISS-NMSSM and the properties of leptonic $g-2$ in Sec. II. We then scan the parameter space that explains both the electron and muon $g-2$ discrepancies and analyze the characteristics of the input parameters and particle mass spectrum in Sec. III. In Sec. IV, we find that our scenario can be embedded into a $\tau$-type sneutrino, which coannihilates with a Higgsino to achieve the observed DM relic density and does not conflict with the current DM direct search observations. In Sec. V, we show the impact of the current LHC SUSY particle direct searches. Finally, we draw conclusions in Sec. VI.

\section{INVERSE SEESAW MECHANISM EXTENDED NEXT-TO-MINIMAL SUPERSYMMETRIC STANDARD MODEL AND THE LEPTON $g-2$}

\section{A. Brief introduction to inverse seesaw next-to-minimal supersymmetric standard model}

The complete definition of the ISS-NMSSM Lagrangian, such as quantum number setting, can be found in Ref. [75]. Here, we only briefly introduce the basic idea of the "inverse-seesaw" extension and the neutrino sector.

The "inverse-seesaw" mechanism is added to the NMSSM framework by introducing two gauge singlet superfields $\hat{\nu}$ and $\hat{X}$ with opposite lepton numbers $L=-1$ and $L=1$, respectively [76]. With the assumptions of $R$-parity conservation, not introducing the $\Delta L=1$ lepton number violation, the superpotential $W$ is given as follows:

$$
\begin{aligned}
W= & Y_{u} \hat{Q} \cdot \hat{H}_{u} \hat{u}+Y_{d} \hat{H}_{d} \cdot \hat{Q} \hat{d}+Y_{e} \hat{H}_{d} \cdot \hat{L} \hat{e}+\lambda \hat{S} \hat{H}_{u} \cdot \hat{H}_{d}+\frac{\kappa}{3} \hat{S}^{3} \\
& +\frac{1}{2} \mu_{X} \hat{X} \hat{X}+\lambda_{N} \hat{S} \hat{\nu} \hat{X}+Y_{\nu} \hat{L} \cdot \hat{H}_{u} \hat{\nu} .
\end{aligned}
$$

The first line of Eq. (2.1) is the standard NMSSM superpotential. The soft breaking terms of the ISS-NMSSM are given as follows 


$$
\begin{aligned}
V_{\text {soft }}= & V_{\mathrm{NMSSM}}+M_{\nu}^{2} \tilde{\nu}_{R} \tilde{\nu}_{R}^{*}+M_{X}^{2} \tilde{x} \tilde{x}^{*} \\
& +\left(\frac{1}{2} B_{\mu_{X}} \tilde{x} \tilde{x}+\lambda_{N} A_{N} S \tilde{\nu}_{R}^{*} \tilde{x}+Y_{\nu} A_{\nu} \tilde{\nu}_{R}^{*} \tilde{L} \cdot H_{u}+\text { H.c. }\right),
\end{aligned}
$$

where $V_{\text {NMSSM }}$ is the NMSSM soft breaking term, and $\tilde{\nu}_{R}$ and $\tilde{x}$ are the scalar parts of superfields $\hat{\nu}$ and $\hat{X}$, respectively. The dimensional parameter $\mu_{X}$ is a small $X$-type neutrino mass term, which often is treated as an effective mass parameter to obtain the tiny masses of active neutrinos. The introduction of $\mu_{X}$ violates the lepton number due to the $\Delta L=2$ term $\mu_{X} \hat{X} \hat{X}$ and the $\mathbb{Z}_{3}$ symmetry of the superpotential.

After the electroweak symmetry breaking, the $9 \times 9$ complex and symmetric neutrino mass matrix $M_{\text {ISS }}$ in the basis $\left(v_{L}, v_{R}^{*}, x\right)$ reads

$$
M_{\mathrm{ISS}}=\left(\begin{array}{ccc}
0 & m_{D}^{T} & 0 \\
m_{D} & 0 & m_{R} \\
0 & m_{R}^{T} & \mu_{X}
\end{array}\right)
$$

where $m_{D}=\frac{1}{\sqrt{2}} Y_{\nu} v_{u}$ and $m_{R}=\frac{1}{\sqrt{2}} \lambda_{N} v_{s}$ are the $3 \times 3$ neutrino Dirac mass matrices. The $M_{\text {ISS }}$ can be diagonalized by a $9 \times 9$ unitary matrix $U_{\nu}$ according to

$$
U_{\nu}^{*} M_{\nu} U_{\nu}^{\dagger}=\operatorname{diag}\left(m_{\nu_{i}}, m_{\nu_{h j}}\right), \quad i=1,2,3, \quad j=1,2, \ldots, 6 .
$$

This gives three active neutrino masses $m_{\nu_{i}}$ and six heavy neutrino masses $m_{\nu_{h j}}$. $M_{\text {ISS }}$ can be diagonalized by block to give the $3 \times 3$ light neutrino mass matrix $M_{\nu}$. Under the inverse seesaw limits $\left\|\mu_{X}\right\| \ll\left\|m_{D}\right\|,\left\|m_{R}\right\|$,

$$
M_{\nu} \simeq m_{D}^{T} m_{R}^{T-1} \mu_{X} m_{R}^{-1} m_{D}
$$

This $M_{\nu}$ is diagonalized by the unitary Pontecorvo-MakiNakagawa-Sakata (PMNS) matrix $U_{\text {PMNS }}$ :

$$
m_{\nu}^{\text {diag }} \equiv \operatorname{diag}\left(m_{\nu_{1}}, m_{\nu_{2}}, m_{\nu_{3}}\right)=U_{\mathrm{PMNS}}^{T} M_{\nu} U_{\mathrm{PMNS}}
$$

In this work, for our phenomenological purpose, we use the $\mu_{X}$-parametrization scheme that was introduced in [77] $]^{1,2}$ to reproduce low-energy neutrino data

\footnotetext{
${ }^{1} \mathrm{~A}$ detailed discussion of $\mu_{X}$ parametrization can also be found in [78]. In principal, $Y_{\nu}$ in the $\mu_{X}$ parametrization can be arbitrarily large except for that the perturbativity of the theory requires $\left|Y_{\nu}\right|^{2} / 4 \pi \leq 1.5$.

${ }^{2}$ The neutrino oscillation data can also be reconstruct by the Casas-Ibarra parametrization, in which the neutrino oscillations are generated by the off-diagonal term in $m_{D}$ [79]. Because of the special role of parameter $Y_{\nu}$ in this work, the $\mu_{X}$ parametrization scheme is more intuitive than the Cassas-Ibarra parametrization scheme.
}

$$
\mu_{X}=m_{R}^{T} m_{D}^{T-1} U_{\mathrm{PMNS}}^{*} m_{\nu}^{\mathrm{diag}} U_{\mathrm{PMNS}}^{\dagger} m_{D}{ }^{-1} m_{R}
$$

$Y_{\nu}$ and $\lambda_{N}$ in this work are assumed to be flavor diagonal, so the neutrino oscillation data is attributed only to the flavor nondiagonal parameter $\mu_{X}$. In this case, the unitary constraint of neutrino sector can be translated into a constraint on the input parameters [74]

$$
\frac{\lambda_{N_{e}}}{Y_{\nu_{e}}} \frac{\mu}{\lambda v_{u}}>14.1, \quad \frac{\lambda_{N_{\mu}}}{Y_{\nu_{\mu}}} \frac{\mu}{\lambda v_{u}}>33.7, \quad \frac{\lambda_{N_{\tau}}}{Y_{\nu_{\tau}}} \frac{\mu}{\lambda v_{u}}>9.4 .
$$

These inequalities indicate that, for given $\lambda_{N}$ and the Higgs sector parameters $\lambda, \tan \beta$, and $\mu$, this unitary constraint allows $Y_{\nu_{e}}$ to be greater than $Y_{\nu_{\mu}}$, which is good for explaining $\Delta a_{e}$.

The ISS mechanism preserve an approximate lepton number conservation. Equation (2.7) indicates that the light neutrino masses require the magnitude of lepton number breaking parameter $\mu_{X}$ to be highly suppressed. Numerically, both $\mu_{X}$ and the soft breaking mass term $B_{\mu_{X}}$ are extremely small $\left(\left|\mu_{X}\right| \lesssim \mathcal{O}(\mathrm{KeV})\right.$ and $\left|B_{\mu_{X}}\right| \lesssim$ $\mathcal{O}\left(100 \mathrm{GeV}^{2}\right)$ ), and they just slightly split the complex sneutrino field into the $C P$-even part and $C P$-odd part. When studying $a_{\ell}$, the influence of these two nonvanishing parameters can be ignored [72]. Because the dimension of the scan parameters is too high, for the sake of simplicity, we simply set the values of $\mu_{X}$ and $B_{\mu_{X}}$ to zero in the following discussion.

The SUSY particles of particular importance to $a_{\ell}$ are sleptons $\tilde{\ell}, \ell$-type sneutrinos, neutralinos $\tilde{\chi}_{i}^{0}$, and charginos $\tilde{\chi}_{i}^{ \pm}$. The neutralino and chargino sector in the ISS-NMSSM were same as that of the NMSSM. On the basis of $\phi^{0}=\left(\tilde{B}, \tilde{W}^{3}, \tilde{H}_{d}^{0}, \tilde{H}_{u}^{0}, \tilde{S}\right)^{T}$, the symmetric neutralino mixing matrix $\mathcal{M}_{0}$ is as follows:

$$
\mathcal{M}^{0}=\left(\begin{array}{ccccc}
M_{1} & 0 & -\frac{g_{1}}{\sqrt{2}} v_{d} & \frac{g_{1}}{\sqrt{2}} v_{u} & 0 \\
& M_{2} & \frac{g_{2}}{\sqrt{2}} v_{d} & -\frac{g_{2}}{\sqrt{2}} v_{u} & 0 \\
& & 0 & -\mu & -\lambda v_{u} \\
& & & 0 & -\lambda v_{d} \\
& & & & 2 \kappa v_{s}
\end{array}\right),
$$

where the Higgsino mass $\mu=\lambda v_{s}$ is an effective $\mu$ term after the electroweak symmetry breaking, and $v_{u}, v_{d}$, and $v_{s}$ represent the vacuum expectation values (VEVs) of the Higgs field $H_{u}, H_{d}$, and $\mathrm{S}$, respectively. The mass eigenstates $\tilde{\chi}_{i}^{0}=N_{i j} \phi_{j}^{0}$ are arranged in ascending order of mass. With the basis $\phi^{+}=\left(\tilde{W}^{+}, \tilde{H}_{u}^{+}\right)$and $\phi^{-}=\left(\tilde{W}^{-}, \tilde{H}_{d}^{-}\right)$, the chargino mass term is given by $\phi^{-} \mathcal{M}^{ \pm} \phi^{+}+$H.c. with the mass matrix

$$
\mathcal{M}^{ \pm}=\left(\begin{array}{cc}
M_{2} & g_{2} v_{u} \\
g_{2} v_{d} & \mu
\end{array}\right)
$$


The corresponding mass eigenstates are defined by

$$
\tilde{\chi}_{i}^{+}=V_{i j} \phi_{j}^{+}, \quad \tilde{\chi}_{i}^{-}=U_{i j} \phi_{j}^{-} .
$$

The symmetric mass matrix $\mathcal{M}_{\tilde{\ell}}^{2}$ for slepton $\tilde{\ell}$ for each flavor $\ell$ in the $\left(\tilde{\ell}_{L}, \tilde{\ell}_{R}\right)$ basis is

$$
\mathcal{M}_{\tilde{\ell}}^{2}=\left(\begin{array}{cc}
M_{L_{\ell}}^{2}+m_{\ell}^{2}+m_{Z}^{2} \cos 2 \beta\left(\sin ^{2} \theta_{W}-\frac{1}{2}\right) & m_{\ell}\left(A_{E_{\ell}}-\mu \tan \beta\right) \\
& M_{E_{\ell}}^{2}-\sin ^{2} \theta_{W} m_{Z}^{2} \cos 2 \beta
\end{array}\right),
$$

and the corresponding rotation matrix is represented by $X^{\ell}$.

In terms of the particle composition, the ISS-NMSSM differs from the NMSSM [80] only in the neutrino sector. For each generation $\ell=e, \mu, \tau$, the sneutrino fields are the mixtures of left-handed sneutrino $\tilde{\nu}_{L}^{\ell}$, right-handed sneutrino $\tilde{\nu}_{R}^{\ell}$, and $x$-type sneutrino $\tilde{\nu}_{x}^{\ell}$. On the basis of $\phi_{\nu}^{\ell}=\left(\tilde{\nu}_{L}^{\ell}, \tilde{\nu}_{R}^{\ell}, \tilde{\nu}_{x}^{\ell}\right)$, the symmetric mass matrix $\mathcal{M}_{\tilde{\nu}}^{2}$ is given by

$$
\mathcal{M}_{\tilde{\nu}}^{2}=\frac{1}{2}\left(\begin{array}{ccc}
2 M_{L_{\ell}}^{2}+Y_{\nu_{\ell}}^{2} v_{u}^{2}+m_{Z}^{2} \cos 2 \beta & Y_{\nu_{\ell}}\left(\sqrt{2} A_{\nu_{\ell}} v_{u}-\lambda v_{d} v_{s}\right) & Y_{\nu_{\ell}} \lambda_{N_{\ell}} v_{u} v_{s} \\
& 2 M_{\nu_{\ell}}^{2}+Y_{\nu_{\ell}}^{2} v_{u}^{2}+\lambda_{N_{\ell}}^{2} v_{s}^{2} & \lambda_{N_{\ell}}\left(\sqrt{2} A_{N_{\ell}} v_{s}+\kappa v_{s}^{2}-\lambda v_{u} v_{d}\right) \\
& & 2 M_{X_{\ell}}^{2}+\lambda_{N_{\ell}}^{2} v_{s}^{2}
\end{array}\right) .
$$

The mass eigenstate of one generation sneutrino is $\tilde{\nu}_{i}^{\ell}=\sum_{j} Z_{i j}^{\ell} \phi_{\nu, j}^{\ell}$, with $Z^{\ell}$ denoting the unitary matrix to diagonalize $\mathcal{M}_{\tilde{\nu}}^{2}$. From Eq. (2.13), one can easily find that the diagonal elements can be adjusted by the soft breaking parameters $M_{L_{\ell}}^{2}, M_{\nu_{\ell}}^{2}$, and $M_{X_{\ell}}^{2}$. For the off-diagonal elements, Higgs VEV terms, such as $Y_{\nu_{\ell}} v_{u}$ and $\lambda_{N_{\ell}} v_{s}$, provide a scale for the mixing of the three fields of lefthanded, right-handed, and $x$-type sneutrinos. The relative signs and the magnitude of different sneutrino components can be adjusted by two $A$-term soft breaking parameters $A_{\nu_{\ell}}$ and $A_{N_{\ell}}$.

\section{B. Lepton anomalous magnetic moment in ISS-NMSSM}

The lepton anomalous magnetic moment $a_{\ell}$ always corresponds to lepton chirality-flipping interactions. In the ISS-NMSSM, the chirality of the $\ell$-lepton number can be flipped by $Y_{e_{\ell}}$ or $Y_{\nu_{\ell}}$. All the SM-like diagrams (the $\ell$-lepton number is carried only by lepton $\ell$ and/or neutrino $\nu_{\ell}$ ) involve only SM particles, so their contribution to $a_{\ell}$ is identical to the SM prediction $a_{\ell}^{\mathrm{SM}}$. Therefore, the SUSY contribution $a_{\ell}^{\mathrm{SUSY}}$, in which the $\ell$-lepton number is carried also by a scalar lepton $\tilde{\ell}$ and/or $\ell$-type sneutrino $\tilde{\nu}_{\ell}$, provides the source of the observed anomaly $\Delta a_{\ell}$.

The general one-loop SUSY contribution to $a_{\ell}$ in the ISS-NMSSM is given as follows [72]:

$$
\begin{aligned}
a_{\ell}^{\mathrm{SUSY}} & =a_{\ell}^{\tilde{\chi}_{\ell}^{\tilde{\ell}}}+a_{\mu}^{\tilde{\chi}^{ \pm} \tilde{\nu}}, \\
a_{\ell}^{\tilde{\chi}^{0} \tilde{\ell}} & =\frac{m_{\ell}}{16 \pi^{2}} \sum_{i, l}\left\{-\frac{m_{\ell}}{12 m_{\tilde{\ell}_{l}}^{2}}\left(\left|n_{i l}^{\mathrm{L}}\right|^{2}+\left|n_{i l}^{\mathrm{R}}\right|^{2}\right) F_{1}^{\mathrm{N}}\left(x_{i l}\right)+\frac{m_{\tilde{\chi}_{i}^{0}}^{0}}{3 m_{\tilde{\ell}_{l}}^{2}} \operatorname{Re}\left(n_{i l}^{\mathrm{L}} n_{i l}^{\mathrm{R}}\right) F_{2}^{\mathrm{N}}\left(x_{i l}\right)\right\}, \\
a_{\ell}^{\tilde{\chi}^{ \pm} \tilde{\nu}} & =\frac{m_{\ell}}{16 \pi^{2}} \sum_{j, n}\left\{\frac{m_{\ell}}{12 m_{\tilde{\nu}_{\ell, n}}^{2}}\left(\left|c_{j n}^{\mathrm{L}}\right|^{2}+\left|c_{j n}^{\mathrm{R}}\right|^{2}\right) F_{1}^{\mathrm{C}}\left(x_{j n}\right)+\frac{2 m_{\tilde{\chi}_{j}^{ \pm}}}{3 m_{\tilde{\nu}_{\ell, n}}^{2}} \operatorname{Re}\left(c_{j n}^{\mathrm{L}} c_{j n}^{\mathrm{R}}\right) F_{2}^{\mathrm{C}}\left(x_{j n}\right)\right\} .
\end{aligned}
$$

Here, $i=1, \ldots, 5$ and $j=1,2$, respectively, denote the neutralino and chargino indices, $l=1,2$ denotes the slepton index, $n=1,2,3$ denotes the sneutrino index, and

$$
\begin{aligned}
n_{i l}^{\mathrm{L}} & =\frac{1}{\sqrt{2}}\left(g_{2} N_{i 2}+g_{1} N_{i 1}\right) X_{l 1}^{\ell, *}-Y_{e_{\ell}} N_{i 3} X_{l 2}^{\ell, *}, \quad n_{i l}^{\mathrm{R}}=\sqrt{2} g_{1} N_{i 1} X_{l 2}^{\ell}+Y_{e_{\ell}} N_{i 3} X_{l 1}^{\ell}, \\
c_{j n}^{\mathrm{L}} & =-g_{2} V_{j 1} Z_{n 1}^{\ell, *}+Y_{\nu_{\ell}} V_{j 2} Z_{n 2}^{\ell, *}, \quad c_{j n}^{\mathrm{R}}=Y_{e_{\ell}} U_{j 2} Z_{n 1}^{\ell} .
\end{aligned}
$$


The kinematic loop function $F(x)$ is normalized with condition $F(1)=1$, which is given as follows:

$$
\begin{aligned}
& F_{1}^{\mathrm{N}}(x)=\frac{2}{(1-x)^{4}}\left(1-6 x+3 x^{2}+2 x^{3}-6 x^{2} \ln x\right), \\
& F_{2}^{\mathrm{N}}(x)=\frac{3}{(1-x)^{3}}\left(1-x^{2}+2 x \ln x\right), \\
& F_{1}^{\mathrm{C}}(x)=\frac{2}{(1-x)^{4}}\left(2+3 x-6 x^{2}+x^{3}+6 x \ln x\right), \\
& F_{2}^{\mathrm{C}}(x)=-\frac{3}{2(1-x)^{3}}\left(3-4 x+x^{2}+2 \ln x\right),
\end{aligned}
$$

with definitions $x_{i l} \equiv m_{\tilde{\chi}_{i}^{0}}^{2} / m_{\tilde{\ell}_{l}}^{2}$ and $x_{j n} \equiv m_{\tilde{\chi}_{j}^{ \pm}}^{2} / m_{\tilde{\nu}_{n}}^{2}$.

Comparing with the MSSM contribution to $a_{\ell}$ given in [81], the ISS-NMSSM contribution contains an extra HS term. As depicted in Fig. 1, it is easy to understand the leading behaviors with the help of diagram that are written in terms of interaction eigenstates, where the insertions of mass and mixing terms and lepton number chirality flips. Accordingly, this HS contribution $a_{\ell}^{\mathrm{HS}}$ can be approximately expressed as

$$
\begin{aligned}
a_{\ell}^{\mathrm{HS}} & \approx m_{\ell}^{2} \times \frac{1}{48 \pi^{2} v} \frac{Y_{\nu_{\ell}}}{\mu \cos \beta}\left\{\sum_{n} Z_{n 1}^{\ell} Z_{n 2}^{\ell} \times x_{n} F_{2}^{C}\left(x_{n}\right)\right\}, \\
x_{n} & \equiv \frac{\mu^{2}}{m_{\tilde{\nu}_{\ell, n}}^{2}},
\end{aligned}
$$

where $v=\sqrt{v_{u}^{2}+v_{d}^{2}}=173 \mathrm{GeV}$, and the function $x F_{2}^{C}(x)$ increases monotonically with $x$, and its value ranging from 0 to 1.5 . Equation (2.17) shows that $a_{\ell}^{\mathrm{HS}}$ is enhanced by the factor $\cos \beta \approx 1 / \tan \beta$ for large $\tan \beta$. A relatively large $Y_{\nu_{\ell}}$ is expected to achieve a larger $a_{\ell}^{\mathrm{HS}}$. The lepton chirality flipping in the HS contribution is reflected in the left-right mixing term $Z_{n 1} Z_{n 2}$ for each sneutrino $\tilde{\nu}_{n}$. If the $x$-fielddominated sneutrino is too heavy, then the contribution

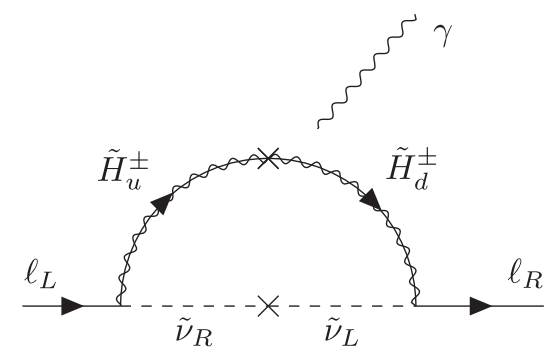

FIG. 1. One-loop diagram of Higgsino-sneutrino contribution, the additional contribution to $a_{\ell}^{\mathrm{SUSY}}$ in the ISS-NMSSM compared to the MSSM. from the left-handed dominated sneutrino and the righthanded dominated state to $a_{\ell}$ cancel each other because $Z_{11}^{\ell} Z_{12}^{\ell} \approx-Z_{21}^{\ell} Z_{22}^{\ell}$. This cancellation can be alleviated by rendering a sizable mixing between $x$ field and the righthanded sneutrino field, which, as shown in Eq. (2.13), can be obtained by choosing a large mixing term $\lambda_{N} v_{s}$. Furthermore, A light $\mu$ is favored for large $a_{\ell}^{\mathrm{HS}}$, so the small $\lambda$ at $\mathcal{O}(0.01)$ often has a larger $a_{\ell}^{\mathrm{HS}}$. This small $\lambda$ is also preferred by the leptonic unitary condition in Eq. (2.8).

Looking back to the MSSM explanation to both anomalies, the first difficulty is that the most of the relevant parameters (except the lepton soft trilinear breaking term $A_{e}$ ) are lepton flavor independent. Specifically, the full expression of $a_{\ell}^{\text {SUSY }}$ in MSSM is very similar to that in ISSNMSSM, and the difference is that $c_{L}$ in Eq. (2.15) of MSSM does not contain the $Y_{\nu_{\ell}} V_{j 2}$ term. The $\mu$ parameter governs the Higgsino $\tilde{H}_{u}-\tilde{H}_{d}$ transition and the dominant part of scalar lepton mixing term, so that the MSSM contribution is proportional to $\mu \tan \beta$. Compared with $\mu \tan \beta, A_{e}$ is usually ignored in the left-handed and right-handed scalar lepton mass mixing. The gaugino mass parameters $M_{1}$ and $M_{2}$ affect the sign of MSSM contribution to $a_{\ell}$. Thus, in general, $a_{\mu}^{\mathrm{SUSY}}$ and $a_{e}^{\mathrm{SUSY}}$ are highly correlated in MSSM. The second difficulty comes from the correlation between $a_{\ell}^{\tilde{\chi}^{0} \tilde{\ell}}$ and $a_{\ell}^{\tilde{\chi}^{ \pm} \tilde{\nu}}$. In MSSM, the mass of the left-handed slepton $\tilde{\ell}_{L}$ is slightly larger than the mass of sneutrino $\tilde{\nu}^{\ell}$ by about $15 \mathrm{GeV}$ for large $\tan \beta$. As a result, the wino-Higgsino loop in $a_{\ell}^{\tilde{\chi}^{0} \tilde{e}}$ is about $-1 / 2$ times that in $a_{\ell}^{\tilde{\chi}^{ \pm} \tilde{\nu}}$.

The most attractive property of the HS term in explaining both anomalies in ISS-NMSSM is that the left-right mixing $Z_{n 1} Z_{n 2}$ is positively related to $A_{\nu}$, which is lepton flavor relevant. The magnitude and sign of the HS contribution can be adjusted by $A_{\nu}$. Numerically, an $\left|A_{\nu}\right|$ at $\mathcal{O}(100 \mathrm{GeV})$ to $\mathcal{O}(1 \mathrm{TeV})$ is sufficient for $a_{\ell}^{\mathrm{HS}}$ to explain both anomalies. Furthermore, the weakening of mass relevance between left-handed dominated slepton $\tilde{\ell}_{L}$ and left-handed dominated sneutrino $\tilde{\nu}_{L}^{\ell}$ gives ISS-NMSSM parameter space more room for interpreting both anomalies.

\section{FEATURES OF COMBINED EXPLANATION TO $\Delta a_{e}$ AND $\Delta a_{\mu}$ IN ISS-NMSSM}

The relevant SUSY particle masses have different contributions to $a_{\ell}^{\text {SUSY }}$. To reveal the detailed features of $a_{\ell}^{\text {SUSY }}$ in the ISS-NMSSM, which is covered up by the complexity of the loop functions $F(x)$ in Eq. (2.16), we use the MultiNest technique $[82,83]$ to scan the ISS-NMSSM parameter space with the following parameter values and ranges: 


$$
\begin{aligned}
& 0<\lambda<0.7, \quad|\kappa|<0.7, \quad 100 \mathrm{GeV}<\mu<500 \mathrm{GeV}, \quad \tan \beta=60 \\
& A_{\lambda}=2 \mathrm{TeV}, \quad-1 \mathrm{TeV}<A_{\kappa}<1 \mathrm{TeV}, \quad-5 \mathrm{TeV}<A_{t}=A_{b}<5 \mathrm{TeV}, \\
& \left|M_{1}\right|<1 \mathrm{TeV}, \quad\left|M_{2}\right|<1.5 \mathrm{TeV}, \\
& 100 \mathrm{GeV}<M_{L_{e}}<1 \mathrm{TeV}, \quad 100 \mathrm{GeV}<M_{E_{e}}<1 \mathrm{TeV}, \quad A_{E_{e}}=0 \\
& 0<Y_{\nu_{e}}<1.0, \quad\left|A_{\nu_{e}}\right|<2 \mathrm{TeV}, \quad 0<\lambda_{N_{e}}<1.0, \quad\left|A_{N_{e}}\right|<2 \mathrm{TeV}, \\
& \left|M_{\nu_{e}}^{2}\right|<1 \mathrm{TeV}^{2}, \quad\left|M_{X_{e}}^{2}\right|<1 \mathrm{TeV}^{2}, \\
& 100 \mathrm{GeV}<M_{L_{\mu}}<1 \mathrm{TeV}, \quad 100 \mathrm{GeV}<M_{E_{\mu}}<1 \mathrm{TeV}, \quad A_{E_{\mu}}=0 \\
& 0<Y_{\nu_{\mu}}<1.0, \quad\left|A_{\nu_{\mu}}\right|<2 \mathrm{TeV}, \quad 0<\lambda_{N_{\mu}}<1.0, \quad\left|A_{N_{\mu}}\right|<2 \mathrm{TeV}, \\
& \left|M_{\nu_{\mu}}^{2}\right|<1 \mathrm{TeV}^{2}, \quad\left|M_{X_{\mu}}^{2}\right|<1 \mathrm{TeV}^{2},
\end{aligned}
$$

where all parameters are defined at the scale of $1 \mathrm{TeV}$. All the other soft breaking parameters, like those first two generation squark, are fixed at a common value of $3 \mathrm{TeV}$. The prior probability distribution function (PDF) of these inputs are set as uniform distributions, and the $n_{\text {live }}$ parameter, which indicates the number of the active points to determine the isolikelihood contour in the MultiNest algorithm iteration, is set at 10000 . The likelihood function $\chi^{2}$ in the scan is taken as

$$
\chi^{2}=\chi_{a_{\ell}}^{2}+\chi_{\mathrm{Higgs}}^{2}+\chi_{\text {veto }}^{2}
$$

where $\chi_{a_{\ell}}^{2}=\frac{1}{2}\left(\frac{a_{e}^{\mathrm{SUSY}}+87 \times 10^{-14}}{36 \times 10^{-14}}\right)^{2}+\frac{1}{2}\left(\frac{a_{\mu}^{\mathrm{SUSY}}-267 \times 10^{-11}}{76 \times 10^{-11}}\right)^{2}$ is a standard Gaussian form of two anomalies. $\chi_{\text {Higgs }}^{2}$ requires the sample in the parameter space given by Eq. (3.1) to predict an SM-like Higgs boson compatible with current experimental observations using the HiggsSignals-2.2.3 code [84-86] and to satisfy the constraints of a direct search of the Higgs boson using the HiggsBounds-5.3.2 code [87]. $\chi_{\text {veto }}^{2}$ is introduced to ensure that the LSP is a Higgsino, the electroweak vacuum is stable, sneutrino fields have not developed nonzero VEVs, and the unitary constraint of Eq. (2.8) is satisfied. If the parameter point satisfies the above assumption, then $\chi_{\text {veto }}^{2}$ is equal to 0 ; otherwise, $\chi_{\text {veto }}^{2}=10000$. Technically, the introduction of $\chi_{\text {veto }}^{2}$ highlights the characteristics of the parameter space of ISSNMSSM explaining the two anomalies, and minimizes the influence of other phenomenological constraints on the statistics of the scanning result. After several repeated scans, we checked that the statistical distribution of the parameters in the results is reproducible.

In the numerical calculation, an ISS-NMSSM model file is generated by the SARAH-4.14.3 package [88,89], the particle spectra of the parameter samples are calculated by the SPheno-4.0.3 [90,91] and FLAVORKIT [92] packages, and electroweak vacuum stability and sneutrino stability are tested by the VEVACIOUs $[93,94]$ package, in which the tunneling time from the input electroweak potential minimum to the true vacuum is obtained via the COSMOTRANSITIONS package [95].

The one-dimensional profile likelihood (PL) plots of $\chi_{a_{e}}^{2}$ with the values normalized to the maximum and marginal posterior PDF of the input parameters are shown in Figs. 2-4. The value of PL for a fixed value of the parameter of interest, $\theta=\theta_{0}$, is the profiled value of nuisance parameters that maximized $\mathcal{L}$. Therefore, in a multidimensional model, the PL can be treated as an indicator of how well the theory can explain the experiments in the subspace of $\theta=\theta_{0}$. Complementarily, the one-dimensional marginal posterior PDF reflects the volume of subspace $\theta=\theta_{0}$.

These figures show that ISS-NMSSM has a large parameter space to give a common explanation of $\Delta a_{e}$ and $\Delta a_{\mu}$. The results are summarized as follows:

(i) In the view of the PL, the smooth bell-shaped PL curves of $a_{e}^{\mathrm{SUSY}}$ and $a_{\mu}^{\mathrm{SUSY}}$ indicate the ability of the ISS-NMSSM to fit the two anomalies. The missing part of the PL curve where $a_{e}^{\mathrm{SUSY}}<-1 \times 10^{-12}$ indicates that a large $a_{e}^{\text {SUSY }}$ is difficult to achieve in theory.

(ii) The distributions of the input parameters $M_{2}, \lambda, \mu$, $Y_{\nu_{e}}, Y_{\nu_{\mu}}$, and $A_{\nu_{e}}$ show some trends that verify the discussion in the previous section. The narrow PL picks of $100 \mathrm{GeV} \lesssim \mu \lesssim 150 \mathrm{GeV}$ and $Y_{\nu_{e}} \geq 0.7$ reflect the properties of Eq. (2.17) and the difficulty of explaining $\Delta a_{e}$. The wider credible region of the $\mu$-type input parameter than that of $e$-type parameter also confirms this feature.

(iii) For most input parameters, the credible regions are smaller than the confidence interval. The PL values are very close to 1 almost in the entire space. This implies that the value of these parameters are not affected by the two anomalies.

(iv) $\mu \sim 110 \mathrm{GeV}$ and $0.015 \lesssim \lambda \lesssim 0.05$ cause the masses of all the singlet Higgs particles to be on the order of several $\mathrm{TeV}$.

(v) $M_{1}$ and $M_{2}$ are often much greater than $\mu$ to reduce the correlations in the MSSM contributions to $a_{e}^{\text {SUSY }}$ 

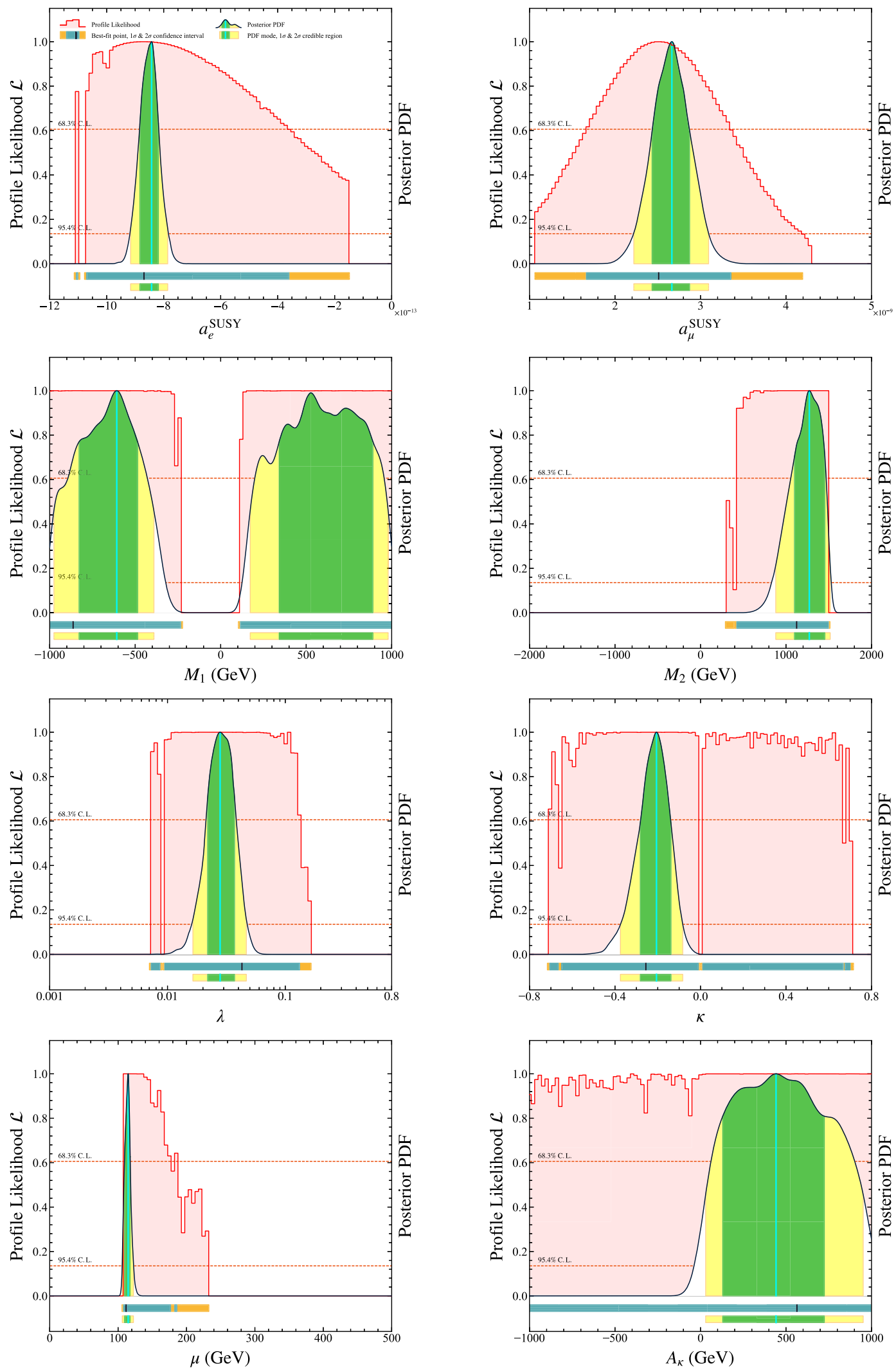

FIG. 2. One-dimensional profile likelihood $\mathcal{L}$ and posterior PDF distributions of $a_{\ell}^{\text {SUSY }}$ and electroweakino input parameters. Regions of orange areas colored blue show the $1 \sigma(2 \sigma)$ confidence interval, and the best point is marked by a black vertical line. Regions of yellow areas colored green represent the $1 \sigma(2 \sigma)$ credible region. 

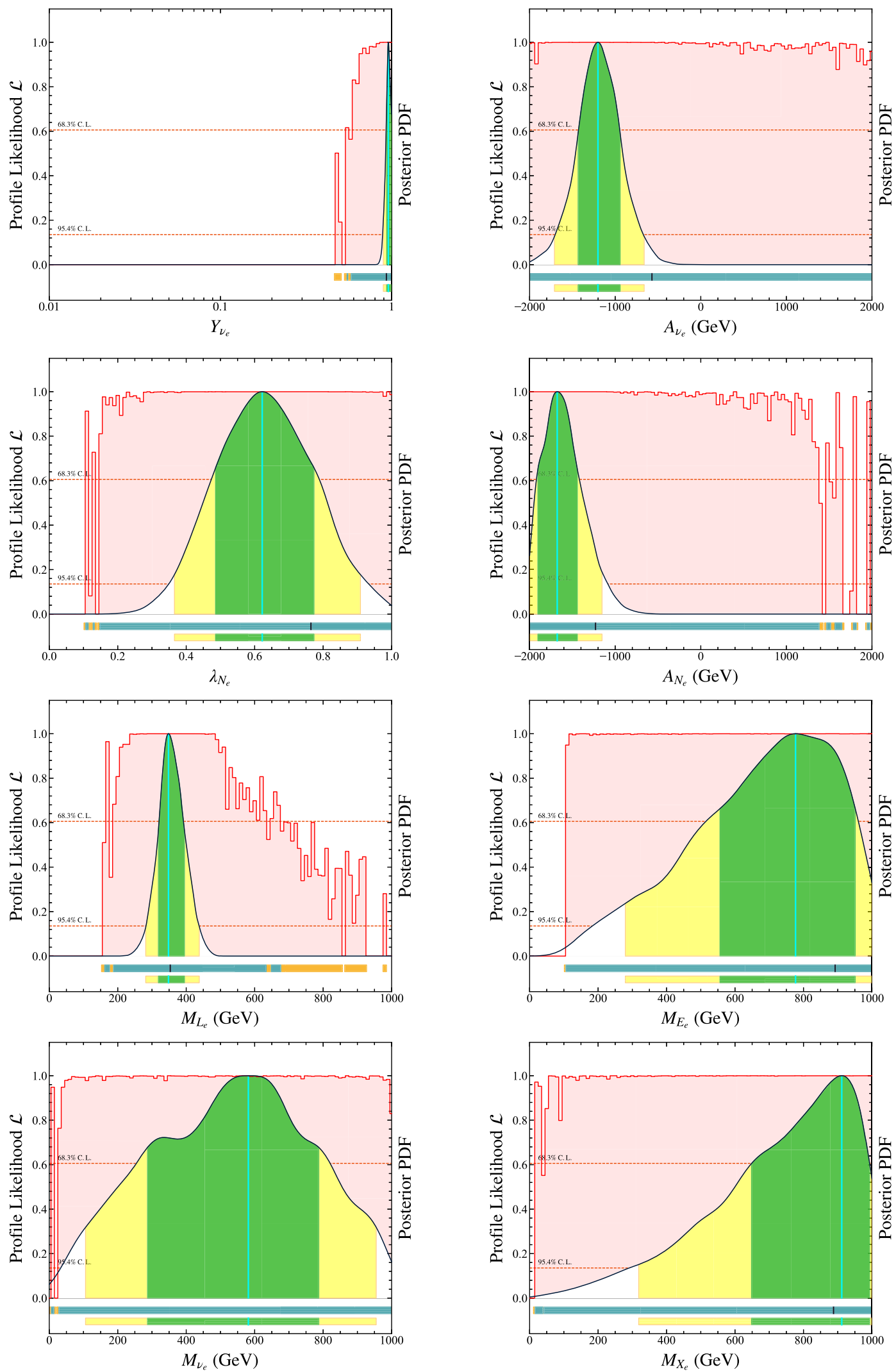

FIG. 3. One-dimensional profile likelihood $\mathcal{L}$ and posterior PDF distributions of the input parameters of $e$-type slepton and sneutrino. Regions of orange areas colored blue show the $1 \sigma(2 \sigma)$ confidence interval, and the best point is marked by a black vertical line. Regions of yellow areas colored green represent the $1 \sigma(2 \sigma)$ credible region. 

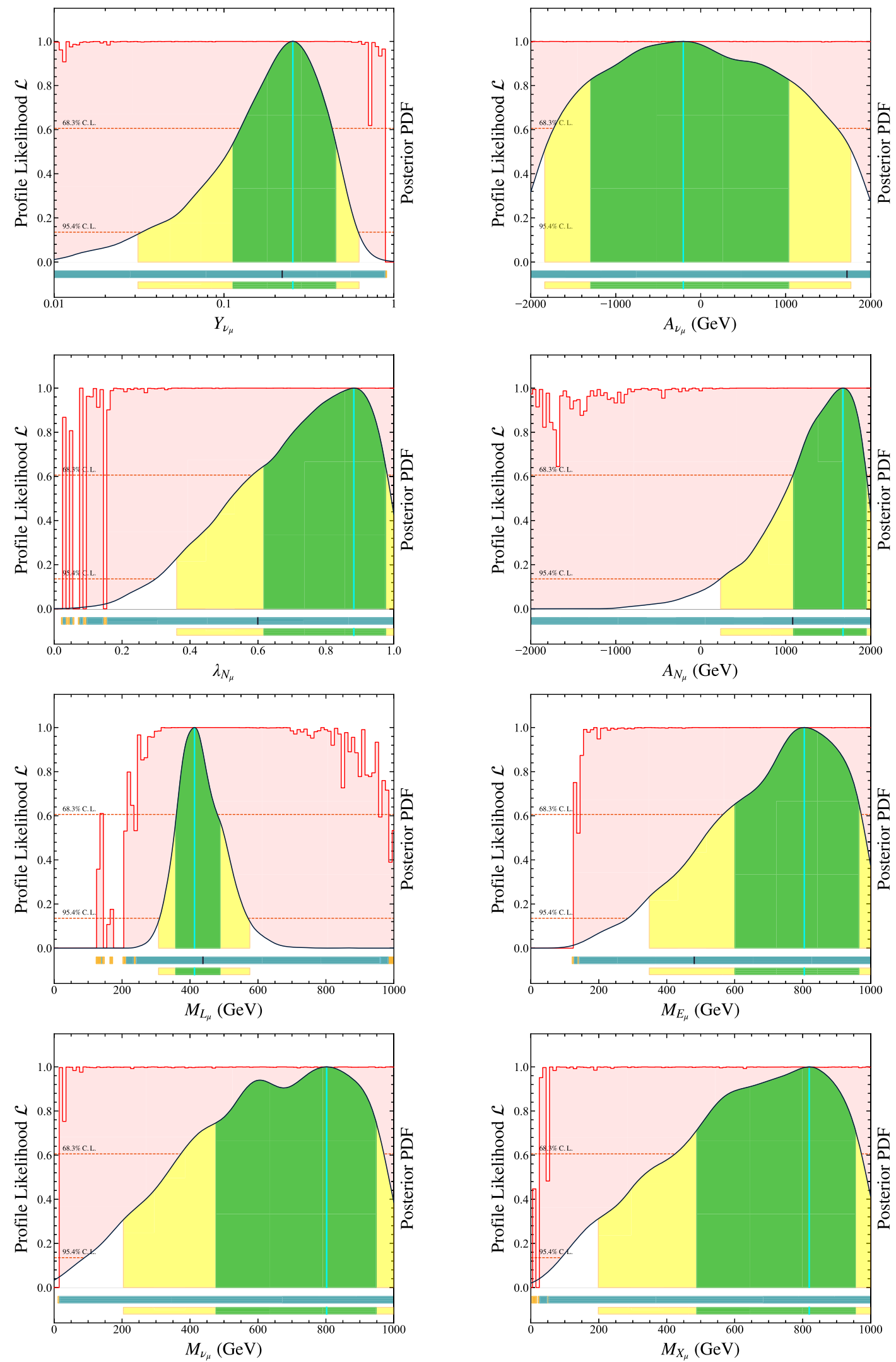

FIG. 4. One-dimensional profile likelihood $\mathcal{L}$ and posterior PDF distributions of the $\mu$-flavor related input parameters. Regions of orange areas colored blue show the $1 \sigma(2 \sigma)$ confidence interval, and the best point is marked by a black vertical line. Regions of yellow areas colored green represent the $1 \sigma(2 \sigma)$ credible region. 


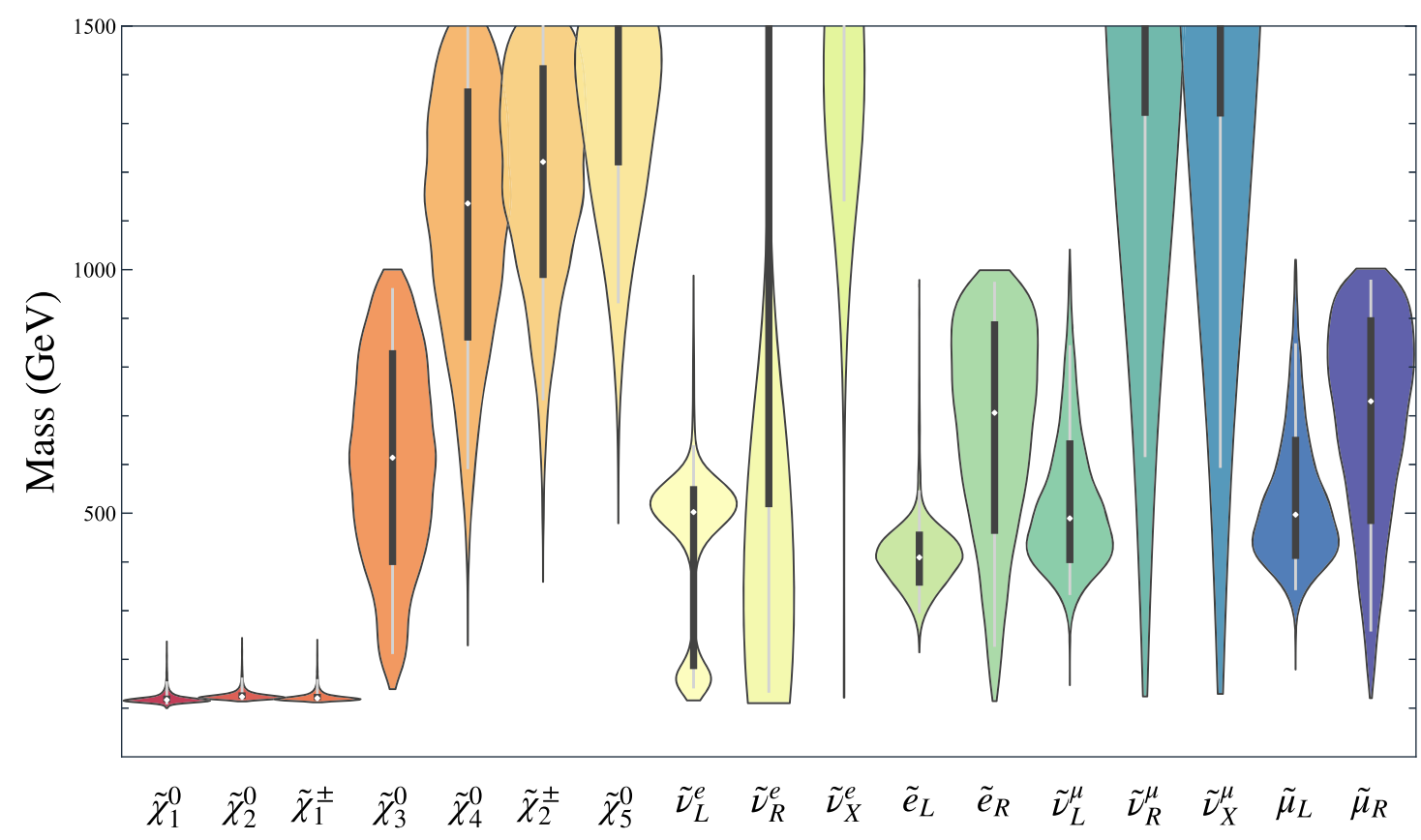

FIG. 5. Violin plots showing the mass distributions of supersymmetry (SUSY) particles. Sleptons and sneutrinos are labeled by their dominated components. The violins are scaled by count. The thick vertical bar in the center indicates the interquartile range with the white dot representing the median, and the long vertical line represents the $95 \%$ confidence interval.
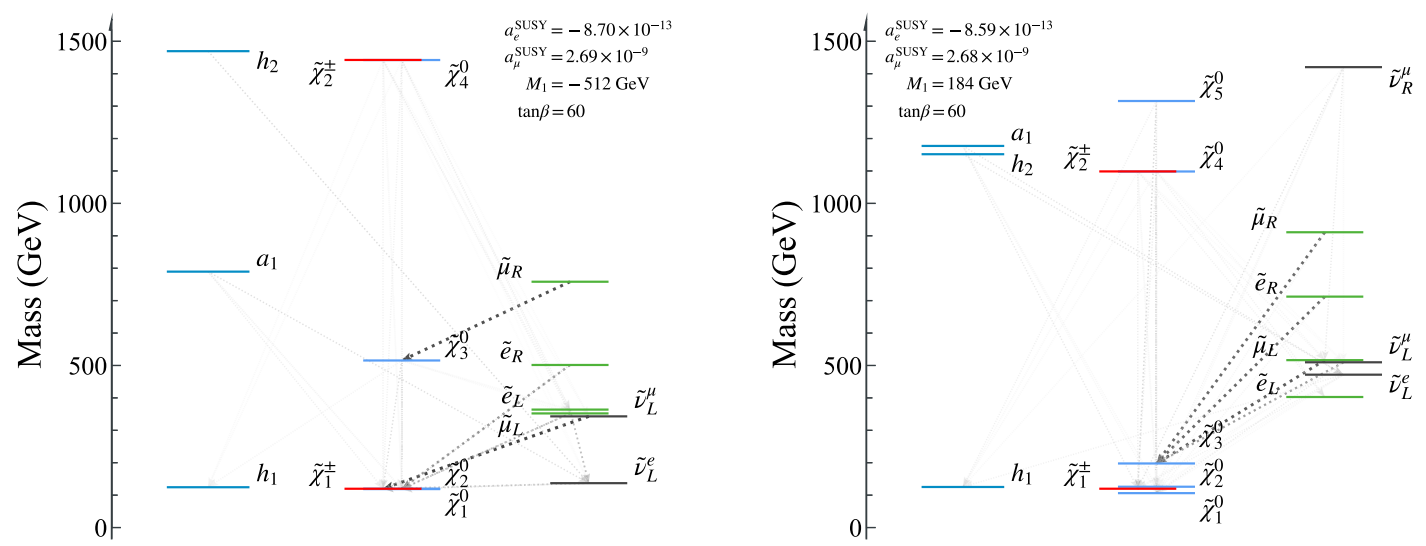

FIG. 6. Higgs and sparticle spectra for the typical samples in the ISS-NMSSM. Sleptons and sneutrinos are labeled by their dominant components, the decay paths are shown with branching ratios $>5 \%$, and the widths of the lines are proportional to the branching ratios.

and $a_{\mu}^{\text {SUSY }}$. The distributions of $M_{1}$ shows that the bino-slepton loop contribution is far from dominant. The distribution of $M_{2}$ shows that the wino-related contributions are suppressed in this explanation.

There were 7989 samples obtained that explained two anomalies within the $2 \sigma$ range in total. In Fig. 5, we plot the mass distributions of the SUSY particles via violin plots, ${ }^{3}$ where sleptons and sneutrinos are labeled by their dominating components. The masses of the Higgsino-dominated triplet states were around $110 \mathrm{GeV}$, and the wino particles

\footnotetext{
${ }^{3}$ A violin plot is similar to a box plot; it shows the probability density smoothed by kernel density estimation [96].
}

were often heavier than $700 \mathrm{GeV}$. The masses of lefthanded selectron $\tilde{e}_{L}$ and $\tilde{\mu}_{L}$ were distributed around $400 \mathrm{GeV}$, and the right-handed sleptons were relatively heavy. In contrast to the MSSM spectrum, the mass of $\tilde{\nu}_{L}^{e}$ can be much lower than that of $\tilde{e}_{L}$ in the ISS-NMSSM.

The Higgs and sparticle spectra for two typical parameter points are shown in Fig. 6. $M_{1}$ may play a crucial role in the mass splitting of Higgsino-dominated neutralinos and chargino. Taking the mixing terms as a perturbation and calculating the neutralino and chargino masses to the first order in perturbation theory, the mass splitting between Higgsino-dominated electroweakinos are approximately given as follows: 


$$
\begin{aligned}
\Delta m\left(\tilde{\chi}_{2}^{0}, \tilde{\chi}_{1}^{0}\right) \approx \mid & \left|\frac{g_{1}^{2} v^{2}\left(M_{1}+\mu \sin 2 \beta\right)}{2\left(M_{1}^{2}-\mu^{2}\right)}+\frac{g_{2}^{2} v^{2}\left(M_{2}+\mu \sin 2 \beta\right)}{2\left(M_{2}^{2}-\mu^{2}\right)}+\frac{\lambda^{2} v^{2}\left(m_{\tilde{S}}-\mu \sin 2 \beta\right)}{m_{\tilde{S}}^{2}-\mu^{2}}\right|, \\
& \approx\left|\frac{g_{1}^{2} v^{2} M_{1}}{2\left(M_{1}^{2}-\mu^{2}\right)}+\frac{g_{2}^{2} v^{2} M_{2}}{2\left(M_{2}^{2}-\mu^{2}\right)}\right|, \\
\Delta m\left(\tilde{\chi}_{1}^{ \pm}, \tilde{\chi}_{1}^{0}\right) \approx & \mid \frac{g_{1}^{2} v^{2}(1+\sin 2 \beta)}{2\left(M_{1}-\mu\right)}+\frac{g_{2}^{2} v^{2}(1+\sin 2 \beta)}{2\left(M_{2}-\mu\right)}+\frac{\lambda^{2} v^{2}(1-\sin 2 \beta)}{m_{\tilde{S}}-\mu} \\
& +\frac{\lambda^{2}\left(g_{1}^{2}+g_{2}^{2}\right) v^{4}}{2\left(M_{1}-\mu\right)\left(M_{\tilde{S}}-\mu\right) \mu}-\frac{g_{2}^{2} v^{2}}{2 \mu}\left(\frac{\left(m_{2} \cos \beta+\mu \sin \beta\right)^{2}}{m_{2}^{2}-\mu^{2}}-\cos ^{2} \beta\right) \mid, \\
\approx & \left|\frac{g_{1}^{2} v^{2}}{2\left(M_{1}-\mu\right)}+\frac{g_{2}^{2} v^{2}}{2\left(M_{2}-\mu\right)}-\frac{g_{2}^{2} v^{2} \mu}{2\left(M_{2}^{2}-\mu^{2}\right)}\right|,
\end{aligned}
$$

where $m_{\tilde{S}}=2 \kappa v_{s}$ is the singlino mass. These formulae indicate that the effect of $M_{1}$ is negligibly small when $\left|M_{1}\right|$ is extremely large, while as it approaches zero from above (below), it can enhance (decrease) the splitting significantly. This characteristic is shown in Fig. 7, where we projected the scanned samples on the $\Delta m\left(\tilde{\chi}_{1}^{ \pm}, \tilde{\chi}_{1}^{0}\right)-$ $\Delta m\left(\tilde{\chi}_{2}^{0}, \tilde{\chi}_{1}^{0}\right)$ plane with the color bar denoting the $M_{1}$ value. This figure reveals that $\Delta m\left(\tilde{\chi}_{1}^{ \pm}, \tilde{\chi}_{1}^{0}\right) \simeq 4 \mathrm{GeV}$ and $\Delta m\left(\tilde{\chi}_{2}^{0}, \tilde{\chi}_{1}^{0}\right) \simeq 10 \mathrm{GeV}$ when $\left|M_{1}\right|=1 \mathrm{TeV}$. When $M_{1} \simeq 200 \mathrm{GeV}$, the mass splittings increase to several tens of $\mathrm{GeV}$, and when $M_{1} \simeq-200 \mathrm{GeV}$, they decrease to less than $1 \mathrm{GeV}$.

\section{DARK MATTER PHENOMENOLOGY}

In the ISS-NMSSM explanation of the two leptonic anomalous magnetic moments $\Delta a_{e}$ and $\Delta a_{\mu}$, the Higgsino mass is less than $200 \mathrm{GeV}$ and acts as the LSP. Such a light Higgsino is not a good DM candidate due to its

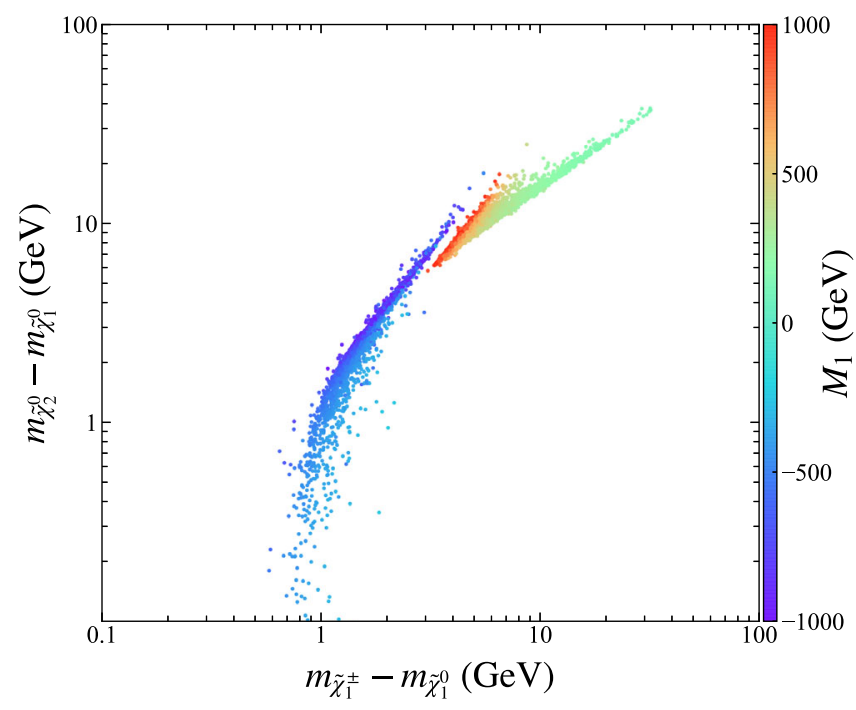

FIG. 7. Mass splitting of Higgsino-dominated neutralinos $\tilde{\chi}_{1,2}^{0}$ and chargino $\tilde{\chi}_{1}^{ \pm}$for the scanned samples with the color bar indicating the value of $M_{1}$. spin-dependent and spin-independent scattering rates with nuclei larger than the current experimental limits [67,78,97-100]. In the ISS-NMSSM, a right-handed-fieldor $x$-field-dominated sneutrino can also serve as the DM candidate. The sizable neutrino Yukawa coupling $Y_{\nu}$ contributes significantly to the DM-nucleon scattering rate, so that $e$-type and $\mu$-type sneutrinos cannot act as DM candidates. Fortunately, $Y_{\nu}$ and $\lambda_{N}$ are chosen to be flavor diagonal, and the flavor mixing effect introduced by $\mu_{X}$ can be ignored. This leaves us room for taking the lightest $\tau$-type sneutrinos, $\tilde{\nu}_{1}^{\tau}$, and its charge conjugate sate, $\tilde{\nu}_{1}^{\tau *}$, as feasible DM candidates [73-75]. ${ }^{4}$ The candidates should be lighter than $\tilde{\chi}_{1}^{0}$ and dominated in components by the righthanded field $\tilde{\nu}_{R}$, the $\tilde{x}$ field, or their mixture. In addition, given the preferred parameters in the previous section, one can further restrict their properties.

\section{A. Sneutrino dark matter}

First, we consider the annihilation of $\tau$-type sneutrinos, whose thermally averaged cross section at the freeze-out temperature should satisfy $\langle\sigma v\rangle_{F} \sim 3 \times 10^{-26} \mathrm{~cm}^{3} / \mathrm{s}$ to obtain the measured abundance. As was pointed out in Ref. [74], the DM has three types of popular annihilation channels:

(i) $\tilde{\nu}_{1}^{\tau} \tilde{\nu}_{1}^{\tau *} \rightarrow h_{s} h_{s}, A_{s} A_{s}$, where $h_{s}$ and $A_{s}$ denote the singlet-dominated $C P$-even and $C P$-odd Higgs bosons, respectively. Since the singlet Higgs dominated particles are much heavier than the DM, these annihilation processes are kinematically forbidden.

(ii) $\tilde{\nu}_{1}^{\tau} \tilde{\nu}_{1}^{\tau *} \rightarrow \bar{\nu}_{h} \nu_{h}, \quad \tilde{\nu}_{1}^{\tau} \tilde{\nu}_{1}^{\tau} \rightarrow \nu_{h} \nu_{h}, \quad$ and $\tilde{\nu}_{1}^{\tau *} \tilde{\nu}_{1}^{\tau *} \rightarrow \bar{\nu}_{h} \bar{\nu}_{h}$, where $\nu_{h}$ represents any of the heavy neutrinos. This process is mainly proceeded via $h_{s}$ in the $s$-channel and/or via the singlino-dominated neutralino in the $t$ channel. Evidently, the annihilation

\footnotetext{
${ }^{4}$ In this study, we take $\tilde{\nu}_{1}^{\tau}$ as a complex field by setting $B_{\nu_{X}}=0$. Consequently, $\tilde{\nu}_{1}^{\tau *}$ also acts as a DM candidate. The ISS-NMSSM is then a two-component DM theory [74]. We add that DM physics requires nontrivial configurations in the theory's parameter space.
} 
cross section is suppressed by the heavy mediator mass.

(iii) Coannihilation with the Higgsinos. In this case, the effective annihilation rate at temperature $T$ takes the following form [101]

$$
\begin{aligned}
\sigma_{\mathrm{eff}}= & \sum_{a, b} \sigma_{a b} \frac{g_{a} g_{b}}{g_{\mathrm{eff}}^{2}}\left(1+\Delta_{a}\right)^{3 / 2}\left(1+\Delta_{b}\right)^{3 / 2} \\
& \times \exp \left[-x\left(\Delta_{a}+\Delta_{b}\right)\right]
\end{aligned}
$$

where $\sigma_{a b}=\sigma(a b \rightarrow X Y), a, b=\tilde{\nu}_{1}^{\tau}, \tilde{\nu}_{1}^{\tau *}, \tilde{\chi}_{1}^{0}, \tilde{\chi}_{2}^{0}, \tilde{\chi}_{1}^{ \pm}$, $X$ and $Y$ denote any possible SM particles, $\Delta_{i} \equiv$ $\left(m_{i}-m_{\tilde{\nu}_{1}^{\tau}}\right) / m_{\tilde{\nu}_{1}^{\tau}}$ for $i=a, b$ represents the mass splitting between the initial particle $i$ and $\tilde{\nu}_{1}^{\tau}$, $x \equiv m_{\tilde{\nu}_{1}^{\tau}} / T, g_{i}$ represents the $i$ th particle's internal degrees of freedom, with $g_{\tilde{\chi}_{i}^{0}}=2, g_{\tilde{\chi}_{1}^{ \pm}}=4$, and $g_{\tilde{\nu}_{1}^{\tau}}=g_{\tilde{\nu}_{1}^{\tau *}}=2$, and the effective degree of freedom $g_{\text {eff }}$ is

$$
g_{\mathrm{eff}} \equiv \sum_{a} g_{a}\left(1+\Delta_{a}\right)^{3 / 2} \exp \left(-x \Delta_{a}\right)
$$

Since the Higgsino pair annihilation cross section is much larger than $3 \times 10^{-26} \mathrm{~cm}^{3} / \mathrm{s}$ for $\mu \simeq 100 \mathrm{GeV}$, these formulas indicate that, even in the case of $\sigma_{\tilde{\nu}_{1}^{\tau} \tilde{\nu}_{1}^{\tau *}} \simeq 0$ and $\sigma_{\tilde{\nu}_{1}^{\tau} \tilde{\nu}_{1}^{\tau}}=\sigma_{\tilde{\nu}_{1}^{\tau} \tilde{\nu}_{1}^{* *}} \simeq 0$, the coannihilation can explain the abundance by adjusting appropriate $\Delta_{i}$ s. For the samples in the Sec. III, we verified that this is feasible by setting $Y_{\nu_{\tau}} \simeq 0, \lambda_{N_{\tau}} \simeq 0$ and adjusting parameters $M_{\nu_{\tau}}^{2}$ and $M_{X_{\tau}}^{2}$ to change $\Delta_{i}$ s. We also found that the measured abundance requires $\Delta m\left(\tilde{\chi}_{1}^{0}, \tilde{\nu}_{1}^{\tau}\right) \sim 5 \mathrm{GeV}$ for $\Delta m\left(\tilde{\chi}_{2}^{0}, \tilde{\chi}_{1}^{0}\right) \simeq 0$ and $\Delta m\left(\tilde{\chi}_{1}^{ \pm}, \tilde{\chi}_{1}^{0}\right) \simeq 1 \mathrm{GeV}$. With the increase in $\Delta m\left(\tilde{\chi}_{2}^{0}, \tilde{\chi}_{1}^{0}\right)$ and $\Delta m\left(\tilde{\chi}_{1}^{ \pm}, \tilde{\chi}_{1}^{0}\right), \Delta m\left(\tilde{\chi}_{1}^{0}, \tilde{\nu}_{1}^{\tau}\right)$ decreases monotonically to maintain the abundance.

Next, we consider the DM-nucleon scattering proceeded by the $t$-channel exchange of the $C P$-even Higgs bosons and $Z$ boson. The spin-dependent cross section is vanishing, and the spin-independent (SI) cross section is [74]

$$
\sigma_{N}^{\mathrm{SI}}=\frac{1}{2}\left(\sigma_{\tilde{\nu}_{1}^{\tau}-N}^{\mathrm{SI}}+\sigma_{\tilde{\nu}_{1}^{\tau *}-N}^{\mathrm{SI}}\right)=\sigma_{N}^{h}+\sigma_{N}^{Z},
$$

where $\sigma_{N}^{h}$ and $\sigma_{N}^{Z}$ with $N=p, n$ denote the Higgs-mediated and the $Z$-mediated contribution, respectively. For the preferred parameters in the last section, $\sigma_{N}^{h}$ and $\sigma_{N}^{Z}$ are approximated by

$$
\begin{aligned}
\sigma_{N}^{h} / \mathrm{cm}^{2} & \simeq 4.2 \times 10^{-44} \times \frac{C_{\tilde{\nu}_{1}^{\tau *} \tilde{\nu}_{1}^{\tau} \Re\left[H_{u}^{0}\right]}^{2}}{m_{\tilde{\nu}_{1}^{\tau}}^{2}}, \\
\sigma_{n}^{Z} / \mathrm{cm}^{2} & =7.4 \times 10^{-39} \times\left(Z_{11}^{\tau}\right)^{4}, \\
\sigma_{n}^{Z} / \mathrm{cm}^{2} & =4.2 \times 10^{-41} \times\left(Z_{11}^{\tau}\right)^{4},
\end{aligned}
$$

where $C_{\tilde{\mathcal{L}}_{1}^{\tau} \tilde{\nu}_{1}^{\tau} \tilde{\nu}_{1} \Re\left[H_{u}^{0}\right]}$ represents the coupling of the DM pair to the $C P$-even $H_{u}^{0}$ field and takes the following form:

$$
\begin{aligned}
C_{\tilde{\nu}_{1}^{*} \tilde{\nu}_{1}^{\tau} \operatorname{Re}\left[H_{u}^{0}\right]} \simeq & -\sqrt{2} \lambda_{N_{\tau}} A_{Y_{\nu_{\tau}}} Z_{11}^{\tau} Z_{12}^{\tau}-\lambda_{N_{\tau}} Y_{\nu_{\tau}} v_{s} Z_{11}^{\tau} Z_{13}^{\tau} \\
& -Y_{\nu_{\tau}}^{2} v_{u} Z_{12}^{\tau} Z_{12}^{\tau} .
\end{aligned}
$$

Noting that $Z_{11}^{\tau}$ is proportional to $Y_{\nu_{\tau}}$, one can conclude that the scattering is suppressed in the case of a small $Y_{\nu_{\tau}}$ and $\lambda_{N_{\tau}}$. This case is favored by current and future DM direct detection experiments [102].

\section{B. Effect of dark matter embedding on sparticle signal at the LHC}

In the ISS-NMSSM, the coupling of the DM to electroweakinos are

$$
\begin{aligned}
g_{\tilde{\nu}_{1}^{\tau} \tau \tilde{\chi}_{i}^{ \pm}}= & Y_{e_{\tau}} U_{i 2}^{*} Z_{11}^{\tau} P_{L}-\left(g_{2} V_{i 1} Z_{11}^{\tau}-Y_{\nu_{\tau}} V_{i 2} Z_{12}^{\tau}\right) P_{R}, \\
g_{\tilde{\nu}_{1}^{\tau} \bar{\nu}_{\tau} \tilde{\chi}_{i}^{0}}= & \frac{1}{\sqrt{2}}\left(g_{1} N_{i 1}-g_{2} N_{i 2}\right) P_{11} Z_{11}^{\tau} P_{R} \\
& -\left(Y_{\nu_{\tau}} N_{i 4} P_{11} Z_{12}^{\tau}+\lambda_{N_{\tau}} N_{i 5} P_{13} Z_{12}^{\tau}\right) P_{R} \\
& -\left(\lambda_{N_{\tau}} N_{i 5}^{*} P_{12}^{*} Z_{13}^{\tau}+Y_{\nu_{\tau}} N_{i 4}^{*} P_{12}^{*} Z_{11}^{\tau}\right) P_{L},
\end{aligned}
$$

where $P$ is a unitary matrix to diagonalize the neutrino mass matrix in one-generation bases $\left(\nu_{L}^{\tau}, \nu_{R}^{\tau}, x^{\tau}\right)$. These expressions show that the coupling strengths are determined by $Y_{\nu_{\tau}}$ and $\lambda_{N_{\tau}}$, and they vanish when $Y_{\nu_{\tau}}=0$. This characteristic has crucial applications in the phenomenology at the LHC. Concretely speaking, in the case where $Y_{\nu_{\tau}}$ and $\lambda_{N_{\tau}}$ are tiny and the DM achieves the measured abundance by the co-annihilation mechanism, the collider signal of the sparticle is roughly identical to that of the NMSSM with $\tilde{\chi}_{1}^{0}$ acting as the LSP [73]. This conclusion can be understood from the following aspects:

(i) The coannihilation mechanism is effective only when $m_{\tilde{\chi}_{1}^{0}} \simeq m_{\tilde{\nu}_{1}^{\tau}}$.

(ii) Since $\tilde{\chi}_{1}^{0}$ of the NLSP decays as $\tilde{\chi}_{1}^{0} \rightarrow \tilde{\nu}_{1}^{\tau} \nu_{\tau}$, it appears as a missing track in the LHC detectors.

(iii) $\tilde{\chi}_{2}^{0}$ may decay by $\tilde{\chi}_{2}^{0} \rightarrow \tilde{\nu}_{1}^{\tau} \nu_{\tau}$ and $\tilde{\chi}_{2}^{0} \rightarrow \tilde{\chi}_{1}^{0} Z^{*} \rightarrow \tilde{\chi}_{1}^{0} f \bar{f}$. The two-body decay is always suppressed by the tiny coupling strength, while the phase space may suppress the three-body decay. We checked that in the case of $Y_{\nu_{\tau}}=\lambda_{N_{\tau}}=10^{-3}$ and $m_{\tilde{\chi}_{2}^{0}}-m_{\tilde{\chi}_{1}^{0}} \lesssim 2 \mathrm{GeV}$, these two decay branching ratios are comparable in size, but the current LHC has no detection capability 
TABLE I. Input parameters, mass spectrum, and decay modes of the sample in Fig. 8 before and after inserting $\tau$-type sneutrino DM.

\begin{tabular}{|c|c|c|c|c|c|}
\hline \multirow{2}{*}{$\frac{\text { Parameters }}{M_{1}}$} & \multirow{2}{*}{$\frac{\text { Value }}{143.2 \mathrm{GeV}}$} & \multirow{2}{*}{$\begin{array}{l}\text { Particles } \\
\tilde{\chi}_{1}^{0}\end{array}$} & \multirow{2}{*}{$\frac{\text { Mass }}{110.5 \mathrm{GeV}}$} & \multicolumn{2}{|c|}{ Before } \\
\hline & & & & Decays & Branching ratio $[\%]$ \\
\hline$M_{2}$ & $650.0 \mathrm{GeV}$ & $\tilde{\chi}_{2}^{0}$ & $-149.4 \mathrm{GeV}$ & $\tilde{\chi}_{1}^{0}(\mathrm{LSP})$ & $\ldots$ \\
\hline$\mu$ & $137.6 \mathrm{GeV}$ & $\tilde{\chi}_{3}^{0}$ & $172.3 \mathrm{GeV}$ & $\tilde{\chi}_{2}^{0} \rightarrow \tilde{\chi}_{1}^{0} q \bar{q} / \tilde{\chi}_{1}^{0} \ell \ell / \tilde{\chi}_{1}^{0} \nu \nu / \tilde{\chi}_{1}^{ \pm} q q^{\prime} / \tilde{\chi}_{1}^{ \pm} \ell \nu$ & $60.67 / 9.29 / 29.44 / 0.20 / 0.39$ \\
\hline$\lambda, \kappa$ & $0.0920,0.6081$ & $\tilde{\chi}_{4}^{0}$ & $686.7 \mathrm{GeV}$ & $\tilde{\chi}_{1}^{ \pm} \rightarrow \tilde{\chi}_{1}^{0} q q^{\prime} / \tilde{\chi}_{1}^{0} \ell \nu$ & $65.38 / 34.72$ \\
\hline$Y_{\nu_{e}}, \lambda_{N_{e}}$ & $0.9565,0.7334$ & $\tilde{\chi}_{5}^{0}$ & $1807 \mathrm{GeV}$ & $\tilde{\chi}_{3}^{0} \rightarrow \tilde{\nu}_{R}^{e} \nu / \tilde{\chi}_{1}^{ \pm} q q^{\prime} / \tilde{\chi}_{1}^{ \pm} \ell \nu$ & $99.87 / 0.07 / 0.04$ \\
\hline$A_{\nu_{e}}$ & $387.5 \mathrm{GeV}$ & $\tilde{\chi}^{ \pm}$ & $140.3 \mathrm{GeV}$ & $\tilde{\chi}_{4}^{0} \rightarrow \tilde{\chi}_{1}^{ \pm} W^{\mp} / \tilde{\chi}_{i}^{0} h / \tilde{\chi}_{i}^{0} Z / \tilde{e}_{L} e / \tilde{\nu} \nu$ & $31.91 / 14.35 / 15.31 / 15.58 / 22.56$ \\
\hline$M_{\ell_{e}}$ & $332.0 \mathrm{GeV}$ & $\tilde{\chi}_{2}^{ \pm}$ & $686.9 \mathrm{GeV}$ & $\tilde{\chi}_{2}^{ \pm} \rightarrow \tilde{\chi}_{1}^{ \pm} h / \tilde{\chi}_{1}^{ \pm} Z / \tilde{\chi}_{i}^{0} W^{ \pm} / \tilde{\nu} \ell / \tilde{e} \nu$ & $14.68 / 15.44 / 31.37 / 22.60 / 15.62$ \\
\hline$M_{E_{e}}$ & $883.5 \mathrm{GeV}$ & $\tilde{e}_{L}$ & $373.5 \mathrm{GeV}$ & Afte & \\
\hline$Y_{\nu_{\mu}}, \lambda_{N_{\mu}}$ & $0.1701,0.8588$ & $\tilde{e}_{R}$ & $859.9 \mathrm{GeV}$ & Decays & Branching ratio $[\%]$ \\
\hline$A_{\nu_{\mu}}$ & $-1943 \mathrm{GeV}$ & $\tilde{\mu}_{L}$ & $767.8 \mathrm{GeV}$ & $\tilde{\chi}_{1}^{0} \rightarrow \tilde{\nu}_{X}^{\tau} \nu$ & 100.0 \\
\hline$M_{\ell_{\mu}}$ & $746.4 \mathrm{GeV}$ & $\tilde{\mu}_{R}$ & $532.2 \mathrm{GeV}$ & $\tilde{\chi}_{2}^{0} \rightarrow \tilde{\chi}_{1}^{0} q \bar{q} / \tilde{\chi}_{1}^{0} \ell \ell / \tilde{\chi}_{1}^{0} \nu \nu / \tilde{\chi}_{1}^{ \pm} q q^{\prime} / \tilde{\chi}_{1}^{ \pm} \ell \nu$ & $57.81 / 8.85 / 32.54 / 0.20 / 0.58$ \\
\hline$M_{E_{\mu}}$ & $569.2 \mathrm{GeV}$ & $\tilde{\nu}_{L}^{e}$ & $462.5 \mathrm{GeV}$ & $\tilde{\chi}_{1}^{ \pm} \rightarrow \tilde{\chi}_{1}^{0} q q^{\prime} / \tilde{\chi}_{1}^{0} \ell \nu$ & $65.38 / 34.72$ \\
\hline$Y_{\nu_{\tau}}, \lambda_{N_{\tau}}$ & $0.0037,0.0368$ & $\tilde{\nu}_{R}^{e}$ & $150.5 \mathrm{GeV}$ & $\tilde{\chi}_{3}^{0} \rightarrow \tilde{\nu}_{R}^{e} \nu / \tilde{\chi}_{1}^{ \pm} q q^{\prime} / \tilde{\chi}_{1}^{ \pm} \ell \nu$ & $99.91 / 0.06 / 0.03$ \\
\hline$Z_{11}^{\tau}$ & $1.112 \times 10^{-5}$ & $\tilde{\nu}_{X}^{e}$ & $1297 \mathrm{GeV}$ & $\tilde{\chi}_{4}^{0} \rightarrow \tilde{\chi}_{1}^{ \pm} W^{\mp} / \tilde{\chi}_{i}^{0} h / \tilde{\chi}_{i}^{0} Z / \tilde{e}_{L} e / \tilde{\nu} \nu$ & $31.89 / 14.34 / 15.30 / 15.57 / 22.60$ \\
\hline$m_{\tilde{\nu}_{X}^{\tau}}$ & $110.3 \mathrm{GeV}$ & $\tilde{\nu}_{L}^{\mu}$ & $757.2 \mathrm{GeV}$ & $\tilde{\chi}_{2}^{ \pm} \rightarrow \tilde{\chi}_{1}^{ \pm} h / \tilde{\chi}_{1}^{ \pm} Z / \tilde{\chi}_{i}^{0} W^{ \pm} / \tilde{\nu} \ell / \tilde{e} \nu$ & $14.67 / 15.44 / 31.35 / 22.64 / 15.61$ \\
\hline$\Omega h^{2}$ & 0.120 & $\tilde{\nu}_{R}^{\mu}$ & $1142 \mathrm{GeV}$ & & \\
\hline$\sigma_{\tilde{\nu}-p}^{\mathrm{SI}}$ & $5.324 \times 10^{-52} \mathrm{~cm}^{2}$ & $\tilde{\nu}_{X}^{\mu}$ & $783.5 \mathrm{GeV}$ & & \\
\hline
\end{tabular}

for such a small mass splitting of Higgsinodominated triplet states [103]. In the case of $m_{\tilde{\chi}_{2}^{0}}-m_{\tilde{\chi}_{1}^{0}}>2 \mathrm{GeV}, \tilde{\chi}_{2}^{0}$ mainly decays by the three-body channel, so its signal is identical to that of the NMSSM with $\tilde{\chi}_{1}^{0}$ as the LSP.

(iv) The situation of $\tilde{\chi}_{1}^{ \pm}$is quite similar to that of $\tilde{\chi}_{2}^{0}$ except that it decays by $\tilde{\chi}_{1}^{ \pm} \rightarrow \tilde{\nu}_{1}^{\tau} \tau$ and $\tilde{\chi}_{1}^{ \pm} \rightarrow$ $\tilde{\chi}_{1}^{0} W^{*} \rightarrow \tilde{\chi}_{1}^{0} f \bar{f}^{\prime}$.

(v) For the other sparticles, their interactions with $\tilde{\nu}_{1}^{\tau}$ are weak, and, thus, their decay chains do not change significantly.

(vi) Due to the constraints of DM direct detection experiments, the values of $Y_{\nu_{\tau}}$ and $\lambda_{N_{\tau}}$ tend to be relatively small. However, in order to satisfy the DM freeze-out mechanism, it is generally believe that the value of $Y_{\nu_{\tau}}$ and $\lambda_{N_{\tau}}$ should be greater than 0.001 . We checked that the lifetime of NLSP $\tilde{\chi}_{1}^{0}$ is always less than $10^{15} \mathrm{GeV}^{-1}$.

Table I shows the details of a sample obtained in the previous section, including the mass spectra and decay modes of some moderately light sparticles. In Fig. 8, we

\footnotetext{
${ }^{5}$ In some models of right-handed sneutrino as LSP [104,105], the stau $\tilde{\tau}_{1}$ decays into the right-handed sneutrino via $\tilde{\tau}_{1} \rightarrow W^{(*)} \tilde{\nu}_{R}$ driven by the tiny neutrino Yukawa coupling $Y_{\nu} \sin \beta \sim 10^{-13}$. Depending on the decay modes and mixing in $\tilde{\tau}$ and $\tilde{\nu}$ sectors, the lifetime of $\tilde{\tau}_{1}$ ranges from a few seconds to over ten years. However, the stau NLSP will not be a long-lived particle in our theory. One reason is that $Y_{\nu_{\tau}}$ should not so small to keep the LSP $\tilde{\nu}_{1}$ in thermal equilibrium in the early universe. Another reason is that the neutralino NLSP is usually predicted by the DM physics, and there is no requirement on the stau mass.
}

show the decay path of these sparticles to illustrate their properties further. This result indicates that the collider signature will not change after being embedded into the DM. Hence a natural way of a $\tau$-type sneutrino DM to achieve the measured relic density is coannihilation with Higgsino. In practice, the Yukawa parameters $Y_{\nu_{\tau}}$ and $\lambda_{N_{\tau}}$ should be small to suppress the DM-nucleon scattering rate, the DM mass $m_{\tilde{\nu}_{1}^{\tau}}$ should be slightly lighter than the Higgsino mass, which is $m_{\tilde{\nu}_{1}^{\tau}} \approx 100 \mathrm{GeV}$.

Since the embedded DM will increase the dimension of the scanning parameters, so we will ignore the DM constraints when studying the collider phenomenology.

\section{CONSTRAINTS FROM LHC SPARTICLE SEARCHES}

Figure 5 shows that to simultaneously explain $\Delta a_{e}$ and $\Delta a_{\mu}$, very light electroweakinos and sleptons are necessary in many samples. Generally, such light sparticles are strongly constrained by the current LHC SUSY searches. In a specific SUSY model, the mass hierarchy and decay modes of sparticles can be significantly different from the simplified model on which the experimental interpretations are based. In this section, we elaborated on the experimental searches we considered, discussed the sparticle decay modes on the ISS-NMSSM parameter space in interpreting $\Delta a_{e}$ and $\Delta a_{\mu}$, and summarized the impact of current LHC sparticle searches on the interpretation.

Using the data taken at $\sqrt{s}=7,8$, and $13 \mathrm{TeV}$, searches for SUSY particles have been conducted for several years. First, we perform analyses at 8 and $13 \mathrm{TeV}$ in SModels v1.2 $[106,107]$ to refine the samples. After this, any point 

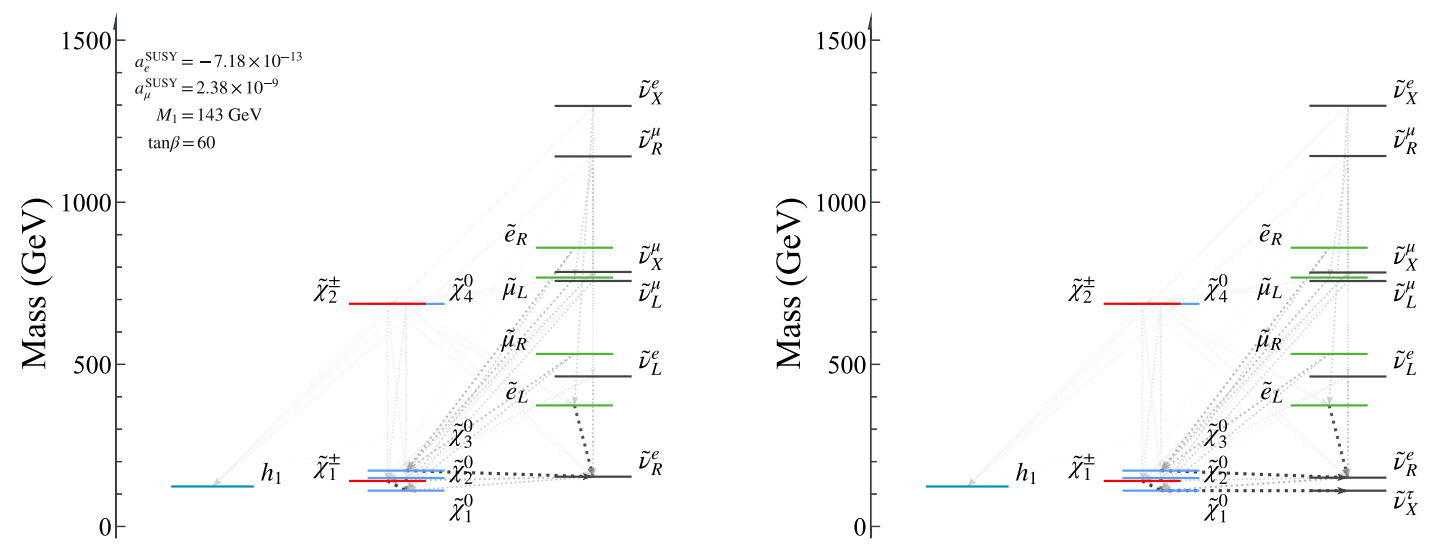

FIG. 8. Higgs and sparticle spectra for the benchmark sample with large Higgsino mass splitting before (left) and after (right) being inserted into $\tau$-type sneutrino dark matter (DM). Sleptons and sneutrinos are labeled by their dominant components, the decay paths are shown with branching ratios $>5 \%$, and the widths of the lines are proportional to the branching ratios.

that passes SModels is further tested by analyses in CheckMATE-2.0.26 [108-110]. The physical processes considered in our work are as follows:

$$
\begin{aligned}
p p & \rightarrow \tilde{\chi}_{i}^{0} \tilde{\chi}_{j}^{ \pm}, \quad i=2,3,4,5, \quad j=1,2, \\
p p & \rightarrow \tilde{\chi}_{i}^{ \pm} \tilde{\chi}_{j}^{\mp}, \quad i=1,2 ; \quad j=1,2, \\
p p & \rightarrow \tilde{\chi}_{i}^{0} \tilde{\chi}_{j}^{0}, \quad i=2,3,4,5 ; \quad j=2,3,4,5, \\
p p & \rightarrow \tilde{\ell}_{i} \tilde{\ell}_{i}, \quad i=e, \mu .
\end{aligned}
$$

The cross section of $\sqrt{s}=8,13 \mathrm{TeV}$ were normalized at the NLO using the PROSPINO2 package [111]. The Monte Carlo events were generated by MadGraph_aMC@NLO $[112,113]$ with the PYTHIA8 package [114] for parton showering and hadronization. The event files were then input into CheckMATE for analysis with DELPHES [115] for detector simulation.

In addition to the analysis that has been implemented before, we added the following newly released LHC analyses to CheckMATE.

(i) ATLAS search for chargino and neutralino production using recursive jigsaw reconstruction in threelepton final states [116]: this analysis is optimized for signals from $\tilde{\chi}_{2}^{0} \tilde{\chi}_{1}^{ \pm}$production with on shell $\mathrm{WZ}$ decay modes. The signal regions (SRs) are split into a low-mass region (jet veto) and the initial state radiation (ISR) region (contains at least one energetic jet) using a variety of kinematic variables, including the dilepton invariant mass $m_{\ell \ell}$, the transverse mass $m_{\mathrm{T}}$, and variables arising from the application of the emulated recursive jigsaw reconstruction technique. The smallness of the mass splittings lead to events with lower- $p_{\mathrm{T}}$ leptons or smaller $E_{\mathrm{T}}^{\text {miss }}$ in the final state. Cuts in the low-mass SR are designed to reduce the $W Z$ background and the number of fake or nonprompt leptons, and cuts in the ISR region requiring large $E_{T}^{\text {miss }}$ to identify events have a real $E_{\mathrm{T}}^{\text {miss }}$ source. This search is sensitive to samples with relative light winos.

(ii) ATLAS search for chargino and slepton pair production in two lepton final states [103]: this analysis targets pair production of charginos and/or sleptons decaying into final states with two electrons or muons. Signal events are required to have an exactly opposite-sign (OS) signal lepton pair with a large invariant mass $m_{\ell \ell}>100 \mathrm{GeV}$ to reduce diboson and $Z+$ jets backgrounds. SRs are separated into same-flavor and different-flavor categories with variables $m_{\ell \ell}$, the stransverse mass $m_{\mathrm{T} 2}$ [117], $E_{\mathrm{T}}^{\text {miss }}$ and $E_{\mathrm{T}}^{\text {miss }}$ significance, and the number of non- $b$-tagged jets. The sensitivity of this analysis to the slepton mass can reach $700 \mathrm{GeV}$, and that to the chargino mass can reach about $1 \mathrm{TeV}(420 \mathrm{GeV})$ of the decay $\operatorname{mode} \tilde{\chi}_{1}^{ \pm} \rightarrow \tilde{\ell} \nu / \ell \tilde{\nu} \rightarrow \ell \nu \tilde{\chi}_{1}^{0}\left(\tilde{\chi}_{1}^{ \pm} \rightarrow W^{ \pm}(\rightarrow \ell \nu) \tilde{\chi}_{1}^{0}\right)$.

(iii) ATLAS search for electroweak production of supersymmetric particles with compressed mass spectra [118]: this was optimized on a simplified model of mass-degenerated Higgsino triplets that assumed $\tilde{\chi}_{2}^{0} \tilde{\chi}_{1}^{ \pm}$production followed by the decays $\tilde{\chi}_{1}^{ \pm} \rightarrow$ $W^{*} \tilde{\chi}_{1}^{0}$ and $\tilde{\chi}_{2}^{0} \rightarrow Z^{*} \tilde{\chi}_{1}^{0}$. It is also sensitive to the degenerate slepton-LSP mass spectrum. The selected events have exactly two OS same-flavor leptons or one lepton plus at least one OS track, and at least one jet is required. The preselection requirements include the requirements that the invariant mass $m_{\ell \ell}$ is derived from the $J / \psi$ meson mass window, that $E_{\mathrm{T}}^{\text {miss }}$ is greater than $120 \mathrm{GeV}$, and that the $p_{\mathrm{T}}$ of the leading jet is larger than $100 \mathrm{GeV}$. After applying the preselection requirements, SRs are further optimized for the specific SUSY scenario into three categories: SR-E (for electroweakino recoiling against ISR), SR-VBF [electroweakino produced through vector boson fusion (VBF)], and SR-S (sleptons recoiling against ISR). A variety of kinematic variables and the 
recursive jigsaw reconstruction technique are used to identify the SUSY signals. Assuming Higgsino production, this search occurs at the minimum mass of $\tilde{\chi}_{2}^{0}$ at $193 \mathrm{GeV}$ at a mass splitting of $9.3 \mathrm{GeV}$.

(iv) ATLAS search for chargino and neutralino pair production in final state with three leptons and missing transverse momentum [119]: this search targets chargino-neutralino pair production decaying via $W Z, W^{*} Z^{*}$, or $W h$ into three-lepton final states. This analysis uses the full LHC run II dataset. The simplified model has an $\tilde{\chi}_{2}^{0}$ mass of up to $640 \mathrm{GeV}$ for on shell $W Z$ decay mode with massless $\tilde{\chi}_{1}^{0}$, up to $300 \mathrm{GeV}$ for the off shell $W Z$ decay mode, and up to $185 \mathrm{GeV}$ for the $W h$ decay mode with an $\tilde{\chi}_{1}^{0}$ mass below $20 \mathrm{GeV}$.

(v) ATLAS search for supersymmetric states with a compressed mass spectrum [120]: this analysis uses the OS lepton pair and large $E_{\mathrm{T}}^{\text {miss }}$, searching for the electroweakino and slepton pair production with a compressed mass spectrum. Two sets of SRs are constructed separately for the production of electroweakinos and sleptons. The electroweakino SRs require the invariant mass of the lepton pair $m_{\ell \ell}$ to be less than $60 \mathrm{GeV}$, and the slepton SRs require the stransverse mass $m_{\mathrm{T} 2}^{m_{\chi}}$ to be greater than $100 \mathrm{GeV}$, where the hypothesized mass of the LSP $m_{\chi}$ is equal to $100 \mathrm{GeV}$. The most sensitive location of the mass splitting is at about $5-10 \mathrm{GeV}$. The $95 \%$ confidence level exclusion limits of the Higgsino, wino, and slepton are up to 145,175 , and $190 \mathrm{GeV}$, respectively.

(vi) CMS combined search for charginos and neutralinos [71]: various simplified models of the SUSY are used in this combined search to interpret the results. Related to our work, the simplified model scenario interpretation of $\tilde{\chi}_{2}^{0} \tilde{\chi}_{1}^{ \pm}$with decays $\tilde{\chi}_{2}^{0} \rightarrow Z^{(*)} \tilde{\chi}_{1}^{0} / h \tilde{\chi}_{1}^{0}$ and $\tilde{\chi}_{1}^{ \pm} \rightarrow W^{(*)} \tilde{\chi}_{1}^{0}$ represents the most stringent constraints from the CMS to date for electroweakino pair production. Compared with the results of individual analyses, this interpretation improves the observed limit in $\tilde{\chi}_{1}^{ \pm}$to about $650 \mathrm{GeV}$ for $W Z$ topology.

(vii) ATLAS search for electroweakino production in $W h$ final states [121]: this was optimized on a simplified model that assumed $\tilde{\chi}_{2}^{0} \tilde{\chi}_{1}^{ \pm}$production with decay modes $\tilde{\chi}_{1}^{ \pm} \rightarrow W^{ \pm} \tilde{\chi}_{1}^{0}$ and $\tilde{\chi}_{2}^{0} \rightarrow h \tilde{\chi}_{1}^{0}$. Signal events were selected with exactly one lepton, two $b$ jets requiring $100 \mathrm{GeV}<m_{b b}<140 \mathrm{GeV}$ and $E_{\mathrm{T}}^{\text {miss }}>240 \mathrm{GeV}$, a transverse mass of the lepton$E_{\mathrm{T}}^{\text {miss }}$ system $m_{\mathrm{T}}$ greater than $100 \mathrm{GeV}$, and a "contransverse mass" $m_{\mathrm{CT}}[122,123]$ greater than $180 \mathrm{GeV}$. Masses of the winos up to $740 \mathrm{GeV}$ are excluded at 95\% confidence level for the massless LSP.

(viii) CMS search in final states with two OS same-flavor leptons, jets, and missing transverse momentum
[124]: this search is sensitive to the on shell and off shell $Z$ boson from BSM processes and to direct slepton production. Search regions are split into on$Z$ SRs, off- $Z$ SRs, and slepton SRs via various kinematic variables, including the invariant mass of the lepton pair $m_{\ell \ell}, M_{\mathrm{T} 2}$, the scalar sum of jet $p_{\mathrm{T}}$, the missing transverse momentum $E_{\mathrm{T}}^{\text {miss }}$, the number of jets $n_{j}$, and the number of $b$-tagging jets $n_{b}$. The result interpretation using the simplified model assuming direct slepton pair production with $100 \%$ decay into dilepton final states shows that the probing limit of the slepton mass $m_{\tilde{\ell}}$ is up to $700 \mathrm{GeV}$. Certainly, this search is sensitive to the sparticles in the ISS-NMSSM interpretation of $\Delta a_{\ell}$. Appendix shows a part of the validation table of the analyses above. We used the $R$ values obtained from CheckMATE to apply the LHC constraints. Here, $R \equiv \max \left\{S_{i} / S_{i, 95}^{\text {obs }}\right\}$ for individual analysis, in which $S_{i}$ represents the simulated event number of the $i$ th SR or bin of the analysis, and $S_{i, 95}^{\text {obs }}$ is the $95 \%$ confidence level upper limit of the event number in the corresponding SR or bin. The combination procedure of the CMS electroweakino search [71] was also performed though the $\mathrm{CL}_{s}$ method [125] with ROOSTATs [126] using the likelihood function described previously [71].

The impacts of LHC constraints on the samples were relatively strong, as 7029 samples were excluded, and only 960 samples survived, corresponding to a total posterior probability of $11.2 \%$. In Fig. 9, we plot the SUSY particle mass of the surviving samples and excluded samples via a split violin plot. ${ }^{6}$ The LHC constraints have not significantly changed the distributions of the SUSY particle mass. In Fig. 10, we plot the samples on the $m_{\tilde{\chi}_{1}^{0}}-\Delta m\left(\tilde{\chi}_{1}^{0}, \tilde{\chi}_{1}^{ \pm}\right)$ plane and the $m_{\tilde{e}_{L}}-m_{\tilde{\mu}_{L}}$ plane with $M_{1}$ valued color. The detection ability of the LHC was mainly affected by the decay modes of SUSY particles.

(1) For the light left-handed slepton pair production processes,

(i) when $\tilde{\nu}_{1}^{e}$ is very light, as shown by the samples in Fig. 8, the dominant decay mode was $\tilde{e}_{L} \rightarrow$ $W \tilde{\nu}_{1}^{e}$ (the sample in Fig. 8 for example), where $\tilde{\nu}_{1}^{e}$ contains large left-handed ingredients. ${ }^{7}$ Such samples are difficult to detect by the current LHC experiments. In the right plane of Figs. 9 and 10 , we find that the $m_{\tilde{e}_{L}}$ values of the surviving samples can reach $200 \mathrm{GeV}$, and such a light $\tilde{e}_{L}$ is good for explaining $\Delta a_{e}$.

\footnotetext{
${ }^{6}$ Similar to a violin plot, the split violin plot splits the violins in half to see the difference between two sample groups. Note that the widths of both sides of the violin are fixed so that the ratio of the two widths does not represent the relative probability or relative number.

${ }^{7}$ There is only one left-handed sneutrino $\tilde{\nu}_{L}^{e}$ in the MSSM or NMSSM, and its mass is slightly lighter than that of the lefthanded selectron $\tilde{e}_{L}$.
} 


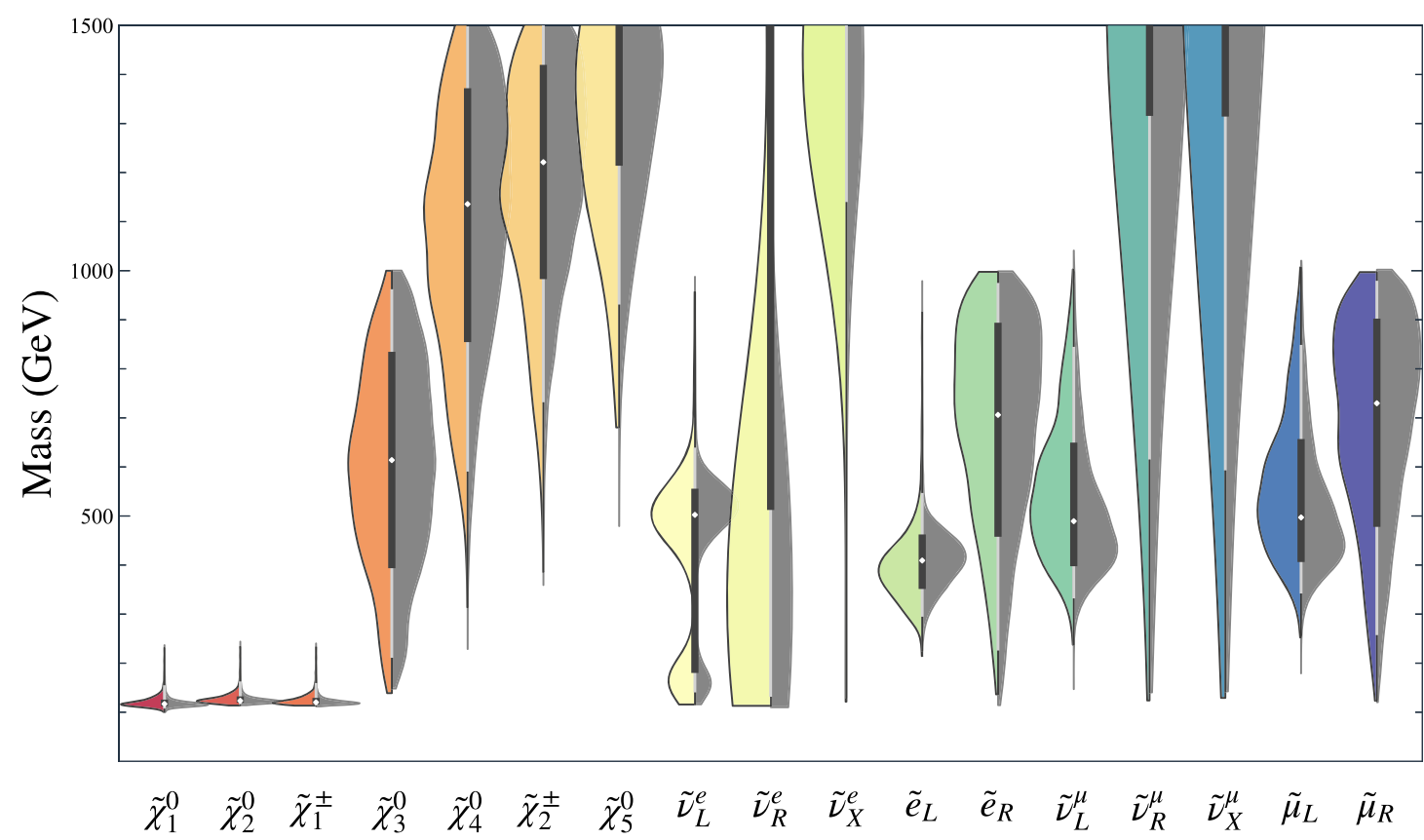

FIG. 9. Split violin plot showing the SUSY particle mass distributions of samples based on the LHC result. The left colorful kernel density estimations (KDEs) are the mass distributions of samples that survive the LHC search results, and the right gray KDEs indicate samples excluded by the LHC results. The medians, interquartile ranges, and 95\% confidence intervals are the same as those in Fig. 5.
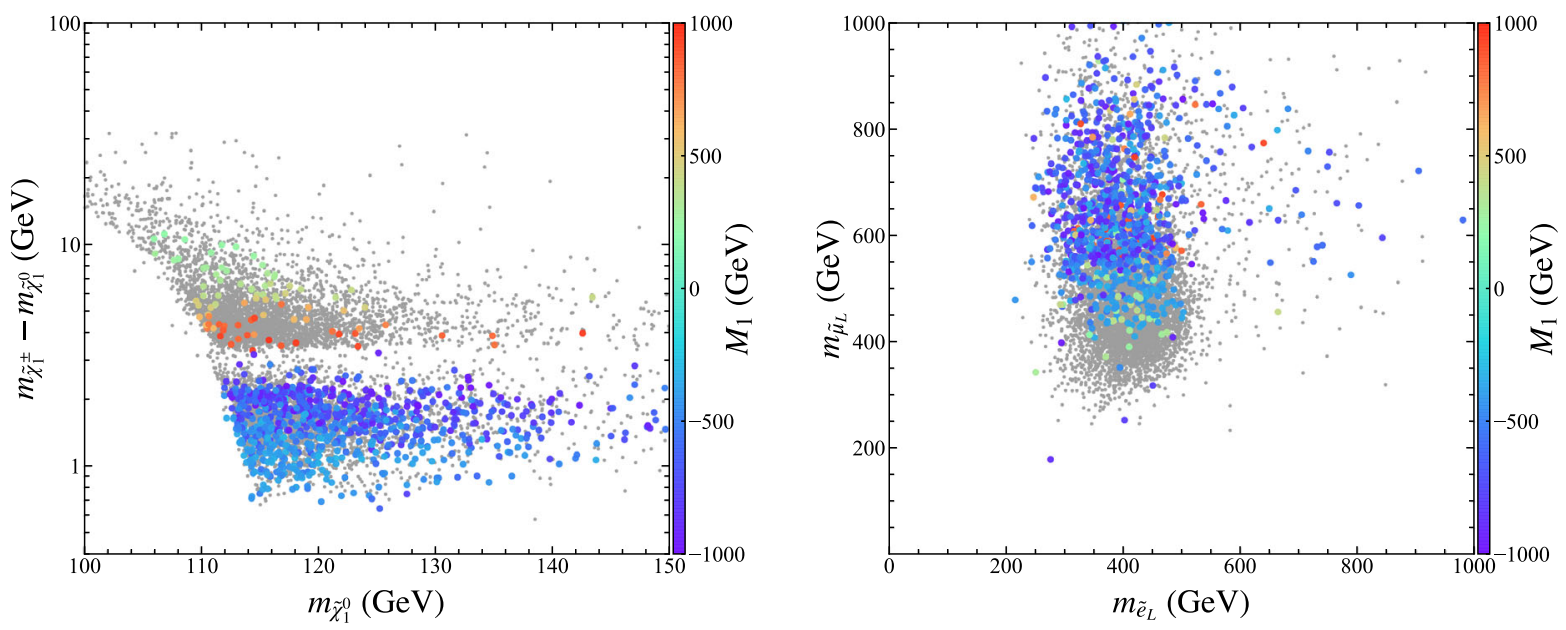

FIG. 10. Samples projected on $m_{\tilde{\chi}_{1}^{0}}-\Delta m\left(\tilde{\chi}_{1}^{0}, \tilde{\chi}_{1}^{ \pm}\right)$plane and $m_{\tilde{e}_{L}}-m_{\tilde{\mu}_{L}}$ plane, where the color indicates the value of $M_{1}$. In each plane, the gray points indicate the samples excluded by the LHC results.

(ii) when $\left|M_{1}\right|$ is lighter than $m_{\tilde{e}_{L}}$ or $m_{\tilde{\nu}_{L}}$, if $\tilde{e}_{L} \rightarrow$ $W \tilde{\nu}_{1}^{e}$ is kinematically forbidden, as shown in the right panel of Fig. 6, the dominated decay mode of $\tilde{\ell}_{L}$ is into $\ell$ plus a binolike neutralino $\tilde{\chi}^{0}$. In this case, the LHC slepton pair production searches provide the most sensitivity to the slepton mass. Figure 10 shows that samples with $\tilde{\mu}_{L}<400 \mathrm{GeV}$ and small $\left|M_{1}\right|$ have difficulty escaping the LHC constraints.

(iii) when $\left|M_{1}\right|$ is very large and $\tilde{\nu}_{1}^{e}$ is very heavy, the left-handed slepton will decay into $\ell \tilde{\chi}_{1}^{0}, \ell \tilde{\chi}_{2}^{0}$, and $\nu \tilde{\chi}_{1}^{ \pm}$, as depicted in the left panel of Fig. 6 . Because the decay products of $\tilde{\chi}_{1}^{ \pm}$are too soft, the LHC constraints for these samples are weaker than those in the light bino case.

(2) For light right-handed slepton pair production processes, the production cross section is about 2.7 times smaller than that of left-handed slepton. The righthanded sleptons mainly decay into $\ell \tilde{\chi}_{1}^{0}$ and $\ell \tilde{\chi}_{2}^{0}$. Only a few samples contain light $\tilde{\mu}_{R}$ or $\tilde{e}_{R}$, so the right-handed slepton had little effect on the result. 
(3) For the Higgsino-dominated electroweakino pair $\tilde{\chi}_{2}^{0} \tilde{\chi}_{1}^{ \pm}$production process, the analysis in Ref. [118] provides a strong constraint on the samples featured by the small positive $M_{1}$, as shown in the left plane of Fig. 10.

(4) For the wino-dominated neutralino/chargino pair production process, the explanation of the two lepton anomalies require $M_{2}>400 \mathrm{GeV}$, and in about more than $97 \%$ samples, the winolike particle mass is greater than $700 \mathrm{GeV}$. Therefore, in this study, because the decay modes of the winos are more complicated, the winos did contribute to the results, but they were not the main effect.

In conclusion, except for some samples where $\tilde{e}_{L}$ decayed to $W \tilde{\nu}_{1}^{e}$, the samples with positive $M_{1}$ were strongly restricted by the current LHC experiments. The searches for the compressed electroweakino spectrum and the searches for sleptons at the LHC are complementary in detecting samples via leptonic final states. Finally, we plot the $a_{e}^{\mathrm{SUSY}}$ and $a_{\mu}^{\mathrm{SUSY}}$ values of the samples in Fig. 11. After considering the constraints from the LHC, there were still a large number of samples that could simultaneously fit $\Delta a_{e}$ and $\Delta a_{\mu}$ at the $1 \sigma$ level.

Before we conclude this section, we briefly comment that the signal of the sparticles may differ significantly from that of the NMSSM in the case of sizable $Y_{\nu_{\tau}}$ and $\lambda_{N_{\tau}}$ [73-75]. In general, because the decay chain becomes lengthened and the signal contains at least two $\tau$ leptons, the sparticles of the ISS-NMSSM are more challenging to detect at the LHC than those of the NMSSM. We drew this conclusion by globally fitting the ISS-NMSSM with various experimental data and studying the scanned samples, similar to our previous work [74]. Therefore, we may overestimate the LHC constraints in this work, but this does not affect the main conclusion that the ISS-NMSSM can easily explain both anomalies.

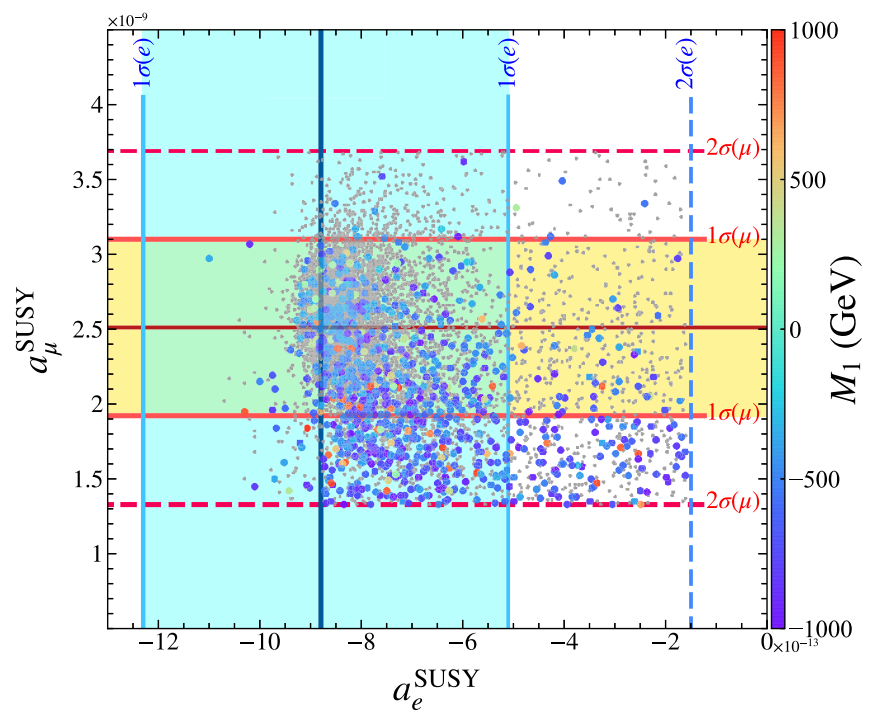

FIG. 11. Similar to Fig. 10 but on $a_{e}^{\text {SUSY }}-a_{\mu}^{\text {SUSY }}$ plane with the $M_{1}$ values represented by the color.
When this work was about to be finished, a new measurement of fine structure constant, via the rubidium atom, was reported [127]:

$$
\alpha^{-1}(\mathrm{Rb})=137.035999206(11),
$$

which leads to a $+1.6 \sigma$ discrepancy of $\Delta a_{e}$ :

$$
\Delta a_{e}(\mathrm{Rb})=a_{e}^{\mathrm{exp}}-a_{e}^{\mathrm{SM}, \mathrm{Rb}}=(48 \pm 30) \times 10^{-14} .
$$

What needs to be emphasized is that there is a $5.4 \sigma$ discrepancy between two measurements $\alpha^{-1}(\mathrm{Cs})$ and $\alpha^{-1}(\mathrm{Rb})$ [127]. It is currently suspected that this difference may be caused by speckle or by a phase shift during the measurement, which requires further study.

Currently, the difference of $\alpha(\mathrm{Rb})$ and $\alpha(\mathrm{Cs})$ are in dispute. We are more concerned about the impacts on the theory. If the future experimental study confirm the correctness of $\alpha(\mathrm{Rb})$, that is there is no significance excess of $a_{e}$ within $2 \sigma$ confidence level, then our previous work [72] shows that in ISS-NMSSM has a large parameter space to explain $\Delta a_{\mu}$. Especially, the HS contribution alone can explain the central value of $\Delta a_{\mu}$, and $\mu$ can be larger than $500 \mathrm{GeV}$ (see the benchmark point in Table II in [72]) correspondingly. On the other hand, if $\alpha(\mathrm{Cs})$ is confirmed in the future, then this work provides a supersymmetric solution without introducing leptonic flavor violation to explain $\Delta a_{e}$ and $\Delta a_{\mu}$ simultaneously.

\section{SUMMARY}

We proposed scenarios in the ISS-NMSSM that explain both electron and muon $g-2$ anomalies without introducing leptonic flavor violation. The results are summarized as follows:

(i) The HS loop induced by the neutrino Yukawa coupling $Y_{\nu}$ plays an important role in the explanation. The advantage of this explanation is that the sign of the HS contribution to $a_{\ell}$ can be determined by the sign of $A_{\nu}$, which greatly reduces the correlation between $a_{e}$ and $a_{\mu}$. A larger HS contribution corresponds to a light $\mu$, which is natural for predicting $m_{Z}$.

(ii) The features of the sparticle spectrum preferentially had large $\tan \beta$, light Higgsino mass $\mu \simeq 110 \mathrm{GeV}$, and heavy wino $M_{2} \gtrsim 600 \mathrm{GeV}$. Moreover, the masses of the left-handed selectron and smuon were around 400 and $500 \mathrm{GeV}$, respectively, and the singlet Higgs-dominated particles are often heavier than $1 \mathrm{TeV} . M_{1}$ affects the mass splitting of Higgsino triplets and the decay mode of the sleptons.

(iii) The mass spectrum can be introduced into a righthanded or $x$-field-dominated $\tau$-type sneutrino as a proper DM candidate, which coannihilated with Higgsinos to obtain the observed DM relic density. As indicated by the previous discussion, choosing $\lambda_{N_{\tau}}$ and $Y_{\nu_{\tau}}$ to be less than about 0.001 can avoid the 
change of the LHC signal caused by the introduction of the DM, and the corresponding DM direct detection cross section is much smaller than the detection ability of the current experiment.

(iv) The signals of the electroweakino/slepton pairs produced at the LHC are sensitive to the parameter space explaining the two anomalies, especially for a positive $M_{1}$. However, due to the compressed mass spectrum, the insensitive decay mode of $\tilde{e}_{L} \rightarrow W \tilde{\nu}_{1}^{e}$, and the very heavy wino, the surviving samples can satisfy the current LHC constraints.

\section{ACKNOWLEDGMENTS}

J.C. and P.Z. wish to thank Jia Liu for the helpful discussion of the experimentally measuring the fine structure constant. This work is supported by the National Natural Science Foundation of China (NNSFC) under Grants No. 11575053, No. 11905044, and No. 12075076.

\section{APPENDIX: VALIDATIONS OF LHC ANALYSES}

This Appendix verifies the correctness of our implementation of the necessary analyses in the package CheckMATE. For the sake of brevity, we only provide

TABLE II. Cut-flow validation of ATLAS analysis [103] for mass point $m\left(\tilde{\chi}_{1}^{ \pm}, \tilde{\chi}_{1}^{0}\right)=(300,50) \mathrm{GeV}$ in search channel of $\tilde{\chi}_{1}^{ \pm} \tilde{\chi}_{1}^{\mp}$ production with $W$-boson-mediated decays. The yields in "Baseline" of "CheckMATE" are normalized to "Baseline" of "ATLAS." "Efficiency" is defined as the ratio of the event number passing though the cut-flow to the event number of the previous event.

\begin{tabular}{|c|c|c|c|c|}
\hline Process & \multicolumn{4}{|c|}{$p p \rightarrow \tilde{\chi}_{1}^{+} \tilde{\chi}_{1}^{-}, \tilde{\chi}_{1}^{ \pm} \rightarrow W^{ \pm} \tilde{\chi}_{1}^{0}$} \\
\hline Point $m\left(\tilde{\chi}_{1}^{ \pm}, \tilde{\chi}_{1}^{0}\right)$ & \multicolumn{4}{|c|}{$(300,50) \mathrm{GeV}$} \\
\hline \multirow[t]{2}{*}{ Generated events } & \multicolumn{4}{|c|}{500,000} \\
\hline & \multicolumn{2}{|c|}{ ATLAS } & \multicolumn{2}{|c|}{ CheckMATE } \\
\hline Selection & Events & Efficiency & Events & Efficiency \\
\hline \multicolumn{5}{|l|}{ Preselection } \\
\hline Baseline & 1144.0 & $\ldots$ & 1144.0 & $\ldots$ \\
\hline Trigger & 793.0 & $69.3 \%$ & 766.0 & $66.9 \%$ \\
\hline OS signal leptons & 661.0 & $83.4 \%$ & 725.1 & $94.7 \%$ \\
\hline$p_{\mathrm{T}}^{\ell_{1}, \ell_{2}}>25 \mathrm{GeV}$ & 565.0 & $85.5 \%$ & 548.6 & $75.7 \%$ \\
\hline$m_{\ell \ell}>25 \mathrm{GeV}$ & 559.0 & $98.9 \%$ & 542.5 & $98.9 \%$ \\
\hline$n_{b-\mathrm{jet}}=0$ & 526.0 & $94.1 \%$ & 507.8 & $93.6 \%$ \\
\hline \multicolumn{5}{|l|}{ SR-DF-0J } \\
\hline Different flavor \& $n_{\text {jets }}=0$ & 122.7 & $23.3 \%$ & 134.3 & $26.4 \%$ \\
\hline$m_{\ell \ell}>100 \mathrm{GeV}$ & 94.2 & $76.8 \%$ & 96.1 & $71.6 \%$ \\
\hline$E_{\mathrm{T}}^{\mathrm{miss}}>110 \mathrm{GeV}$ & 46.5 & $49.4 \%$ & 43.4 & $45.2 \%$ \\
\hline$E_{\mathrm{T}}^{\text {miss }}$ significance $>10$ & 42.2 & $90.8 \%$ & 39.5 & $90.9 \%$ \\
\hline$m_{\mathrm{T} 2}>100 \mathrm{GeV}$ & 26.4 & $62.6 \%$ & 26.3 & $66.7 \%$ \\
\hline \multicolumn{5}{|l|}{ SR-DF-1J } \\
\hline Different flavor \& $n_{\text {jets }}=1$ & 81.9 & $15.6 \%$ & 84.6 & $16.7 \%$ \\
\hline$m_{\ell \ell}>100 \mathrm{GeV}$ & 62.3 & $76.1 \%$ & 57.7 & $68.2 \%$ \\
\hline$E_{\mathrm{T}}^{\mathrm{miss}}>110 \mathrm{GeV}$ & 33.8 & $54.3 \%$ & 30.3 & $52.5 \%$ \\
\hline$E_{\mathrm{T}}^{\mathrm{miss}}$ significance $>10$ & 27.2 & $80.5 \%$ & 28.2 & $93.3 \%$ \\
\hline$m_{\mathrm{T} 2}>100 \mathrm{GeV}$ & 15.3 & $56.3 \%$ & 14.7 & $52.0 \%$ \\
\hline \multicolumn{5}{|l|}{ SR-SF-0J } \\
\hline Same flavor $\& n_{\text {jets }}=0$ & 138.7 & $26.4 \%$ & 111.1 & $21.9 \%$ \\
\hline$m_{\ell \ell}>121.2 \mathrm{GeV}$ & 92.4 & $75.3 \%$ & 74.2 & $66.8 \%$ \\
\hline$E_{\mathrm{T}}^{\mathrm{miss}}>110 \mathrm{GeV}$ & 47.1 & $50.0 \%$ & 40.4 & $54.5 \%$ \\
\hline$E_{\mathrm{T}}^{\text {miss }}$ significance $>10$ & 42.9 & $92.3 \%$ & 35.9 & $88.7 \%$ \\
\hline$m_{\mathrm{T} 2}>100 \mathrm{GeV}$ & 25.4 & $61.9 \%$ & 21.8 & $60.7 \%$ \\
\hline \multicolumn{5}{|l|}{ SR-SF-1J } \\
\hline Same flavor $\& n_{\text {jets }}=1$ & 88.8 & $16.9 \%$ & 82.4 & $16.2 \%$ \\
\hline$m_{\ell \ell}>121.2 \mathrm{GeV}$ & 58.9 & $66.3 \%$ & 48.0 & $58.3 \%$ \\
\hline$E_{\mathrm{T}}^{\mathrm{miss}}>110 \mathrm{GeV}$ & 32.6 & $55.4 \%$ & 25.4 & $52.8 \%$ \\
\hline$E_{\mathrm{T}}^{\mathrm{miss}}$ significance $>10$ & 26.9 & $82.5 \%$ & 24.9 & $98.0 \%$ \\
\hline$m_{\mathrm{T} 2}>100 \mathrm{GeV}$ & 14.0 & $52.0 \%$ & 11.2 & $45.1 \%$ \\
\hline
\end{tabular}


validation of the latest analyses. Table II shows the cut-flow validation of the analysis in Ref. [103] for chargino pair production channel. Table III shows the cut-flow validation for the slepton pair production channel of analysis [118]. All the cut-flow data were provided by experimental groups. The results indicate that our simulations were in good agreement with the analysis of the experimental groups.

TABLE III. Similar to Table II, but for the cut-flow validation of ATLAS analysis [116] in the search channel of the slepton pair production with mass point $m\left(\tilde{\ell}, \tilde{\chi}_{1}^{0}\right)=(150,140) \mathrm{GeV}$.

\begin{tabular}{|c|c|c|c|c|}
\hline Process & \multicolumn{4}{|c|}{$p p \rightarrow \tilde{\ell} \tilde{\ell}, \tilde{\ell} \rightarrow \ell \tilde{\chi}_{1}^{0}$} \\
\hline Point & \multicolumn{4}{|c|}{$m_{\tilde{\ell}}=150 \mathrm{GeV} ; m_{\tilde{\chi}_{1}^{0}}=140 \mathrm{GeV}$} \\
\hline \multirow[t]{2}{*}{ Generated events } & \multicolumn{4}{|c|}{100,000} \\
\hline & \multicolumn{2}{|c|}{ ATLAS } & \multicolumn{2}{|c|}{ CheckMATE } \\
\hline Selection & Events & Efficiency & Events & Efficiency \\
\hline$E_{\mathrm{T}}^{\text {miss }}$ trigger & 2355.37 & $\cdots$ & 2355.37 & $\cdots$ \\
\hline 3rd lepton veto & 1014.55 & $43.1 \%$ & 1079.07 & $45.8 \%$ \\
\hline $3 \mathrm{GeV}<m_{\ell \ell}<3.2 \mathrm{GeV}$ veto & 1013.21 & $99.9 \%$ & 1077.69 & $99.9 \%$ \\
\hline Lepton author 16 veto & 1009.48 & $99.6 \%$ & 1077.69 & $100.0 \%$ \\
\hline $\min \left(\Delta \phi\left(\right.\right.$ jet,$\left.\left.p_{\mathrm{T}}^{\text {miss }}\right)\right)>0.4$ & 970.36 & $96.1 \%$ & 1049.11 & $97.4 \%$ \\
\hline$\Delta \phi\left(j_{1}, p_{\mathrm{T}}^{\mathrm{miss}}\right)>2.0$ & 961.15 & $99.1 \%$ & 1027.05 & $97.9 \%$ \\
\hline Lepton truth matching & 958.99 & $99.8 \%$ & 1027.05 & $100.0 \%$ \\
\hline $1 \mathrm{GeV}<m_{\ell \ell}<60 \mathrm{GeV}$ & 827.86 & $86.3 \%$ & 883.55 & $86.0 \%$ \\
\hline$\Delta R_{e e}>0.3, \Delta R_{\mu \mu}>0.05, \Delta R_{e \mu}>0.2$ & 826.19 & $99.8 \%$ & 883.48 & $99.9 \%$ \\
\hline$p_{\mathrm{T}}^{\ell_{1}}>5 \mathrm{GeV}$ & 823.70 & $99.7 \%$ & 880.95 & $99.7 \%$ \\
\hline$n_{\text {jets }} \geq 1$ & 810.59 & $98.4 \%$ & 880.95 & $100.0 \%$ \\
\hline$p_{\mathrm{T}}^{j_{1}}>100 \mathrm{GeV}$ & 705.86 & $87.1 \%$ & 702.58 & $79.8 \%$ \\
\hline$n_{b \text {-jets }}=0$ & 611.05 & $86.6 \%$ & 643.78 & $91.6 \%$ \\
\hline$m_{\tau \tau}<0$ or $m_{\tau \tau}>160 \mathrm{GeV}$ & 533.29 & $87.3 \%$ & 569.78 & $88.5 \%$ \\
\hline Same flavor & 532.33 & $99.8 \%$ & 569.01 & $99.9 \%$ \\
\hline \multicolumn{5}{|l|}{ SR-highMass } \\
\hline$E_{\mathrm{T}}^{\text {miss }}>200 \mathrm{GeV}$ & 229.81 & $43.2 \%$ & 265.83 & $46.7 \%$ \\
\hline $\max \left(0.85,0.98-0.02 \times m_{\mathrm{T} 2}^{100}\right)<R_{\mathrm{ISR}}<1.0$ & 160.30 & $69.8 \%$ & 165.78 & $62.4 \%$ \\
\hline$p_{\mathrm{T}}^{\ell_{2}}>\min \left(20.0,2.5+2.5 \times\left(m_{T_{2}}^{100}-100\right)\right)$ & 70.71 & $44.1 \%$ & 72.51 & $43.7 \%$ \\
\hline$m_{\mathrm{T} 2}^{100}<140 \mathrm{GeV}$ & 70.71 & $100.0 \%$ & 72.51 & $100.0 \%$ \\
\hline$m_{\mathrm{T} 2}^{100}<130 \mathrm{GeV}$ & 70.71 & $100.0 \%$ & 72.51 & $100.0 \%$ \\
\hline$m_{\mathrm{T} 2}^{100}<120 \mathrm{GeV}$ & 70.71 & $100.0 \%$ & 72.31 & $99.7 \%$ \\
\hline$m_{\mathrm{T} 2}^{100}<110 \mathrm{GeV}$ & 70.71 & $100.0 \%$ & 72.23 & $99.9 \%$ \\
\hline$m_{\mathrm{T} 2}^{100}<105 \mathrm{GeV}$ & 53.72 & $76.0 \%$ & 57.10 & $79.1 \%$ \\
\hline$m_{\mathrm{T} 2}^{100}<102 \mathrm{GeV}$ & 20.21 & $37.6 \%$ & 23.77 & $41.6 \%$ \\
\hline$m_{\mathrm{T} 2}^{100}<101 \mathrm{GeV}$ & 9.38 & $46.4 \%$ & 9.90 & $41.6 \%$ \\
\hline$m_{\mathrm{T} 2}^{120}<100.5 \mathrm{GeV}$ & 4.68 & $49.9 \%$ & 4.86 & $49.1 \%$ \\
\hline \multicolumn{5}{|l|}{ SR-lowMass } \\
\hline $150 \mathrm{GeV}<E_{\mathrm{T}}^{\text {miss }}<200 \mathrm{GeV}$ & 146.36 & $27.5 \%$ & 167.63 & $29.5 \%$ \\
\hline $0.8<R_{\mathrm{ISR}}<1.0$ & 107.82 & $73.7 \%$ & 93.17 & $55.6 \%$ \\
\hline$p_{\mathrm{T}}^{\ell_{2}}>\min \left(15.0,7.5+0.75 \times\left(m_{T_{2}}^{100}-100\right)\right)$ & 52.74 & $48.9 \%$ & 42.29 & $45.4 \%$ \\
\hline$m_{\mathrm{T} 2}^{100}<140 \mathrm{GeV}$ & 52.74 & $100.0 \%$ & 42.29 & $100.0 \%$ \\
\hline$m_{\mathrm{T} 2}^{100}<130 \mathrm{GeV}$ & 52.74 & $100.0 \%$ & 42.29 & $100.0 \%$ \\
\hline$m_{\mathrm{T} 2}^{100}<120 \mathrm{GeV}$ & 52.74 & $100.0 \%$ & 42.29 & $100.0 \%$ \\
\hline$m_{\mathrm{T} 2}^{100}<110 \mathrm{GeV}$ & 52.64 & $99.8 \%$ & 41.65 & $98.5 \%$ \\
\hline$m_{\mathrm{T} 2}^{100}<105 \mathrm{GeV}$ & 38.05 & $72.3 \%$ & 29.09 & $69.9 \%$ \\
\hline$m_{\mathrm{T} 2}^{100}<102 \mathrm{GeV}$ & 16.66 & $43.8 \%$ & 11.24 & $38.6 \%$ \\
\hline$m_{\mathrm{T} 2}^{100}<101 \mathrm{GeV}$ & 8.70 & $52.2 \%$ & 5.60 & $49.8 \%$ \\
\hline$m_{\mathrm{T} 2}^{120}<100.5 \mathrm{GeV}$ & 4.39 & $50.5 \%$ & 2.29 & $40.9 \%$ \\
\hline
\end{tabular}


[1] J.S. Schwinger, On quantum electrodynamics and the magnetic moment of the electron, Phys. Rev. 73, 416 (1948).

[2] R. H. Parker, C. Yu, W. Zhong, B. Estey, and H. Müller, Measurement of the fine-structure constant as a test of the Standard Model, Science 360, 191 (2018).

[3] T. Aoyama, T. Kinoshita, and M. Nio, Revised and improved value of the QED tenth-order electron anomalous magnetic moment, Phys. Rev. D 97, 036001 (2018).

[4] D. Hanneke, S. Hoogerheide, and G. Gabrielse, Cavity control of a single-electron quantum cyclotron: Measuring the electron magnetic moment, Phys. Rev. A 83, 052122 (2011).

[5] D. Hanneke, S. Fogwell, and G. Gabrielse, New Measurement of the Electron Magnetic Moment and the Fine Structure Constant, Phys. Rev. Lett. 100, 120801 (2008).

[6] P. Zyla et al. (Particle Data Group Collaboration), Review of particle physics, Prog. Theor. Exp. Phys. 2020, 083C01 (2020).

[7] T. Aoyama, M. Hayakawa, T. Kinoshita, and M. Nio, Complete Tenth-Order QED Contribution to the Muon g-2, Phys. Rev. Lett. 109, 111808 (2012).

[8] T. Aoyama, T. Kinoshita, and M. Nio, Theory of the anomalous magnetic moment of the electron, Atoms 7, 28 (2019).

[9] A. Czarnecki, W. J. Marciano, and A. Vainshtein, Refinements in electroweak contributions to the muon anomalous magnetic moment, Phys. Rev. D 67, 073006 (2003).

[10] C. Gnendiger, D. Stöckinger, and H. Stöckinger-Kim, The electroweak contributions to $(g-2)_{\mu}$ after the Higgs boson mass measurement, Phys. Rev. D 88, 053005 (2013).

[11] M. Davier, A. Hoecker, B. Malaescu, and Z. Zhang, Reevaluation of the hadronic vacuum polarisation contributions to the Standard Model predictions of the muon $g-2$ and $\alpha\left(m_{Z}^{2}\right)$ using newest hadronic cross-section data, Eur. Phys. J. C 77, 827 (2017).

[12] A. Keshavarzi, D. Nomura, and T. Teubner, Muon $g-2$ and $\alpha\left(M_{Z}^{2}\right)$ : a new data-based analysis, Phys. Rev. D 97, 114025 (2018).

[13] G. Colangelo, M. Hoferichter, and P. Stoffer, Two-pion contribution to hadronic vacuum polarization, J. High Energy Phys. 02 (2019) 006.

[14] M. Hoferichter, B.-L. Hoid, and B. Kubis, Three-pion contribution to hadronic vacuum polarization, J. High Energy Phys. 08 (2019) 137.

[15] M. Davier, A. Hoecker, B. Malaescu, and Z. Zhang, A new evaluation of the hadronic vacuum polarisation contributions to the muon anomalous magnetic moment and to $\boldsymbol{\alpha}\left(\mathbf{m}_{\mathbf{Z}}^{\mathbf{2}}\right)$, Eur. Phys. J. C 80, 241 (2020).

[16] A. Keshavarzi, D. Nomura, and T. Teubner, The $g-2$ of charged leptons, $\alpha\left(M_{Z}^{2}\right)$ and the hyperfine splitting of muonium, Phys. Rev. D 101, 014029 (2020).

[17] A. Kurz, T. Liu, P. Marquard, and M. Steinhauser, Hadronic contribution to the muon anomalous magnetic moment to next-to-next-to-leading order, Phys. Lett. B 734, 144 (2014).

[18] K. Melnikov and A. Vainshtein, Hadronic light-by-light scattering contribution to the muon anomalous magnetic moment revisited, Phys. Rev. D 70, 113006 (2004).
[19] P. Masjuan and P. Sánchez-Puertas, Pseudoscalar-pole contribution to the $\left(g_{\mu}-2\right)$ : A rational approach, Phys. Rev. D 95, 054026 (2017).

[20] G. Colangelo, M. Hoferichter, M. Procura, and P. Stoffer, Dispersion relation for hadronic light-by-light scattering: Two-pion contributions, J. High Energy Phys. 04 (2017) 161.

[21] M. Hoferichter, B.-L. Hoid, B. Kubis, S. Leupold, and S. P. Schneider, Dispersion relation for hadronic light-by-light scattering: Pion pole, J. High Energy Phys. 10 (2018) 141.

[22] A. Gérardin, H. B. Meyer, and A. Nyffeler, Lattice calculation of the pion transition form factor with $N_{f}=2+1$ Wilson quarks, Phys. Rev. D 100, 034520 (2019).

[23] J. Bijnens, N. Hermansson-Truedsson, and A. RodríguezSánchez, Short-distance constraints for the HLbL contribution to the muon anomalous magnetic moment, Phys. Lett. B 798, 134994 (2019).

[24] G. Colangelo, F. Hagelstein, M. Hoferichter, L. Laub, and P. Stoffer, Longitudinal short-distance constraints for the hadronic light-by-light contribution to $(g-2)_{\mu}$ with large$N_{c}$ Regge models, J. High Energy Phys. 03 (2020) 101.

[25] T. Blum, N. Christ, M. Hayakawa, T. Izubuchi, L. Jin, C. Jung, and C. Lehner, The Hadronic Light-By-Light Scattering Contribution to the Muon Anomalous Magnetic Moment from Lattice QCD, Phys. Rev. Lett. 124, 132002 (2020).

[26] G. Colangelo, M. Hoferichter, A. Nyffeler, M. Passera, and P. Stoffer, Remarks on higher-order hadronic corrections to the muon $g-2$, Phys. Lett. B 735, 90 (2014).

[27] B. Abi et al. (Muon g-2 Collaboration), Measurement of the Positive Muon Anomalous Magnetic Moment to 0.46 ppm, Phys. Rev. Lett. 126, 141801 (2021).

[28] G. Bennett et al. (Muon g-2 Collaboration), Final report of the muon E821 anomalous magnetic moment measurement at BNL, Phys. Rev. D 73, 072003 (2006).

[29] T. Aoyama et al., The anomalous magnetic moment of the muon in the Standard Model, Phys. Rep. 887, 1 (2020).

[30] A. Crivellin, M. Hoferichter, and P. Schmidt-Wellenburg, Combined explanations of $(g-2)_{\mu, e}$ and implications for a large muon EDM, Phys. Rev. D 98, 113002 (2018).

[31] E. J. Chun and T. Mondal, Explaining $g-2$ anomalies in two Higgs doublet model with vector-like leptons, J. High Energy Phys. 11 (2020) 077.

[32] S. Jana, V. P. K., W. Rodejohann, and S. Saad, Dark matter assisted lepton anomalous magnetic moments and neutrino masses, Phys. Rev. D 102, 075003 (2020).

[33] C. Arbeláez, R. Cepedello, R. M. Fonseca, and M. Hirsch, $(g-2)$ anomalies and neutrino mass, Phys. Rev. D 102, 075005 (2020).

[34] I. Doršner, S. Fajfer, and S. Saad, $\mu \rightarrow e \gamma$ selecting scalar leptoquark solutions for the $(g-2)_{e, \mu}$ puzzles, Phys. Rev. D 102, 075007 (2020).

[35] F. J. Botella, F. Cornet-Gomez, and M. Nebot, Electron and muon $g-2$ anomalies in general flavour conserving two Higgs doublets models, Phys. Rev. D 102, 035023 (2020).

[36] B. Dutta, S. Ghosh, and T. Li, Explaining $(g-2)_{\mu, e}$, KOTO anomaly and MiniBooNE excess in an extended Higgs model with sterile neutrinos, Phys. Rev. D 102, 055017 (2020). 
[37] C. Hati, J. Kriewald, J. Orloff, and A. Teixeira, Anomalies in ${ }^{8} \mathrm{Be}$ nuclear transitions and $(g-2)_{e, \mu}$ : towards a minimal combined explanation, J. High Energy Phys. 07 (2020) 235.

[38] J.-L. Yang, T.-F. Feng, and H.-B. Zhang, Electron and muon $(g-2)$ in the B-LSSM, J. Phys. G 47, 055004 (2020).

[39] C.-H. Chen and T. Nomura, Electron and muon $g-2$, radiative neutrino mass, and $\ell^{\prime} \rightarrow \ell \gamma$ in a $U(1)_{e-\mu}$ model, Nucl. Phys. B964, 115314 (2021).

[40] L. Calibbi, M. López-Ibáñez, A. Melis, and O. Vives, Muon and electron $g-2$ and lepton masses in flavor models, J. High Energy Phys. 06 (2020) 087.

[41] S. Jana, P. K. Vishnu, and S. Saad, Resolving electron and muon $g-2$ within the 2HDM, Phys. Rev. D 101, 115037 (2020).

[42] N. Haba, Y. Shimizu, and T. Yamada, Muon and electron $g-2$ and the origin of fermion mass hierarchy, Prog. Theor. Exp. Phys. 2020, 093 B05 (2020).

[43] A. Cárcamo Hernández, S. King, H. Lee, and S. Rowley, Is it possible to explain the muon and electron $g-2$ in a $Z^{\prime}$ model?, Phys. Rev. D 101, 115016 (2020).

[44] M. Badziak and K. Sakurai, Explanation of electron and muon $\mathrm{g}-2$ anomalies in the MSSM, J. High Energy Phys. 10 (2019) 024.

[45] M. Bauer, M. Neubert, S. Renner, M. Schnubel, and A. Thamm, Axionlike Particles, Lepton-Flavor Violation, and a New Explanation of $a_{\mu}$ and $a_{e}$, Phys. Rev. Lett. 124, 211803 (2020).

[46] M. Abdullah, B. Dutta, S. Ghosh, and T. Li, $(g-2)_{\mu, e}$ and the ANITA anomalous events in a three-loop neutrino mass model, Phys. Rev. D 100, 115006 (2019).

[47] M. Endo and W. Yin, Explaining electron and muon $g-2$ anomaly in SUSY without lepton-flavor mixings, J. High Energy Phys. 08 (2019) 122.

[48] A. Crivellin and M. Hoferichter, Combined explanations of $(g-2)_{\mu}$, $(g-2)_{e}$ and implications for a large muon EDM, Proc. Sci., ALPS2019 (2020) 009 [arXiv:1905.03789].

[49] B. Dutta and Y. Mimura, Electron $g-2$ with flavor violation in MSSM, Phys. Lett. B 790, 563 (2019).

[50] H. Davoudiasl and W. J. Marciano, Tale of two anomalies, Phys. Rev. D 98, 075011 (2018).

[51] X.-F. Han, T. Li, L. Wang, and Y. Zhang, Simple interpretations of lepton anomalies in the lepton-specific inert two-Higgs-doublet model, Phys. Rev. D 99, 095034 (2019).

[52] A. E. C. Hernández, S. F. King, and H. Lee, Fermion mass hierarchies from vector-like families with an extended 2HDM and a possible explanation for the electron and muon anomalous magnetic moments, Phys. Rev. D 103, 115024 (2021).

[53] L. Delle Rose, S. Khalil, and S. Moretti, Explaining electron and muon $g-2$ anomalies in an aligned 2-Higgs doublet model with right-handed neutrinos, Phys. Lett. B 816, 136216 (2021).

[54] S.-P. Li, X.-Q. Li, Y.-Y. Li, Y.-D. Yang, and X. Zhang, Power-aligned 2HDM: A correlative perspective on $(g-2)_{e, \mu}$, J. High Energy Phys. 01 (2021) 034.
[55] C.-K. Chua, Data-driven study of the implications of anomalous magnetic moments and lepton flavor violating processes of $e, \mu$ and $\tau$, Phys. Rev. D 102, 055022 (2020).

[56] M. Endo, S. Iguro, and T. Kitahara, Probing $e \mu$ flavorviolating ALP at Belle II, J. High Energy Phys. 06 (2020) 040 .

[57] G. Hiller, C. Hormigos-Feliu, D. F. Litim, and T. Steudtner, Anomalous magnetic moments from asymptotic safety, Phys. Rev. D 102, 071901 (2020).

[58] S. Gardner and X. Yan, Light scalars with lepton number to solve the $(g-2)_{e}$ anomaly, Phys. Rev. D 102, 075016 (2020).

[59] J. Aebischer, W. Dekens, E. E. Jenkins, A. V. Manohar, D. Sengupta, and P. Stoffer, Effective field theory interpretation of lepton magnetic and electric dipole moments, arXiv:2102.08954.

[60] A. Bodas, R. Coy, and S. J. D. King, Solving the electron and muon $g-2$ anomalies in $Z^{\prime}$ models, arXiv:2102.07781.

[61] J. Liu, C. E. M. Wagner, and X.-P. Wang, A light complex scalar for the electron and muon anomalous magnetic moments, J. High Energy Phys. 03 (2019) 008.

[62] I. Bigaran and R. R. Volkas, Getting chirality right: Single scalar leptoquark solutions to the $(g-2)_{e, \mu}$ puzzle, Phys. Rev. D 102, 075037 (2020).

[63] H. Banerjee, B. Dutta, and S. Roy, Supersymmetric gauged $\mathrm{U}(1)_{L_{\mu}-L_{\tau}}$ model for electron and muon $(g-2)$ anomaly, J. High Energy Phys. 03 (2021) 211.

[64] C. Cornella, P. Paradisi, and O. Sumensari, Hunting for ALPs with Lepton Flavor Violation, J. High Energy Phys. 01 (2020) 158.

[65] G. Giudice, P. Paradisi, and M. Passera, Testing new physics with the electron g-2, J. High Energy Phys. 11 (2012) 113.

[66] H. Baer, V. Barger, S. Salam, D. Sengupta, and X. Tata, The LHC higgsino discovery plane for present and future SUSY searches, Phys. Lett. B 810, 135777 (2020).

[67] J. Cao, Y. He, L. Shang, Y. Zhang, and P. Zhu, Current status of a natural NMSSM in light of LHC $13 \mathrm{TeV}$ data and XENON-1T results, Phys. Rev. D 99, 075020 (2019).

[68] S. Dimopoulos and G. Giudice, Naturalness constraints in supersymmetric theories with nonuniversal soft terms, Phys. Lett. B 357, 573 (1995).

[69] G. Giudice and R. Rattazzi, Living dangerously with lowenergy supersymmetry, Nucl. Phys. B757, 19 (2006).

[70] H. Baer, V. Barger, P. Huang, and X. Tata, Natural supersymmetry: LHC, dark matter and ILC searches, J. High Energy Phys. 05 (2012) 109.

[71] A. Sirunyan et al. (CMS Collaboration), Combined search for electroweak production of charginos and neutralinos in proton-proton collisions at $\sqrt{s}=13 \mathrm{TeV}$, J. High Energy Phys. 03 (2018) 160.

[72] J. Cao, J. Lian, L. Meng, Y. Yue, and P. Zhu, Anomalous muon magnetic moment in the inverse seesaw extended next-to-minimal supersymmetric standard model, Phys. Rev. D 101, 095009 (2020).

[73] J. Cao, L. Meng, Y. Yue, H. Zhou, and P. Zhu, Suppressing the scattering of WIMP dark matter and nucleons in supersymmetric theories, Phys. Rev. D 101, 075003 (2020). 
[74] J. Cao, Y. He, Y. Pan, Y. Yue, H. Zhou, and P. Zhu, Impact of leptonic unitarity and dark matter direct detection experiments on the NMSSM with inverse seesaw mechanism, J. High Energy Phys. 12 (2020) 023.

[75] J. Cao, X. Guo, Y. He, L. Shang, and Y. Yue, Sneutrino DM in the NMSSM with inverse seesaw mechanism, J. High Energy Phys. 10 (2017) 044.

[76] A. Abada, G. Bhattacharyya, D. Das, and C. Weiland, A possible connection between neutrino mass generation and the lightness of a NMSSM pseudoscalar, Phys. Lett. B 700, 351 (2011).

[77] E. Arganda, M. J. Herrero, X. Marcano, and C. Weiland, Imprints of massive inverse seesaw model neutrinos in lepton flavor violating Higgs boson decays, Phys. Rev. D 91, 015001 (2015).

[78] H. Baer, V. Barger, and H. Serce, SUSY under siege from direct and indirect WIMP detection experiments, Phys. Rev. D 94, 115019 (2016).

[79] J. A. Casas and A. Ibarra, Oscillating neutrinos and $\mu \rightarrow e, \gamma$, Nucl. Phys. B618, 171 (2001).

[80] U. Ellwanger, C. Hugonie, and A. M. Teixeira, The nextto-minimal supersymmetric standard model, Phys. Rep. 496, 1 (2010).

[81] S. P. Martin and J. D. Wells, Muon anomalous magnetic dipole moment in supersymmetric theories, Phys. Rev. D 64, 035003 (2001).

[82] F. Feroz, M. Hobson, and M. Bridges, MultiNest: An efficient and robust Bayesian inference tool for cosmology and particle physics, Mon. Not. R. Astron. Soc. 398, 1601 (2009).

[83] F. Feroz, M. Hobson, E. Cameron, and A. Pettitt, Importance nested sampling and the MultiNest algorithm, Open J. Astrophys. 2, 10 (2019).

[84] P. Bechtle, S. Heinemeyer, O. Stål, T. Stefaniak, and G. Weiglein, Higgssignals: Confronting arbitrary Higgs sectors with measurements at the Tevatron and the LHC, Eur. Phys. J. C 74, 2711 (2014).

[85] O. Stål and T. Stefaniak, Constraining extended Higgs sectors with HiggsSignals, Proc. Sci., EPS-HEP2013 (2013) 314.

[86] P. Bechtle, S. Heinemeyer, O. Stål, T. Stefaniak, and G. Weiglein, Probing the standard model with Higgs signal rates from the tevatron, the LHC and a future ILC, J. High Energy Phys. 11 (2014) 039.

[87] P. Bechtle, O. Brein, S. Heinemeyer, O. Stål, T. Stefaniak, G. Weiglein, and K. E. Williams, HiggsBounds - 4: Improved tests of extended Higgs sectors against exclusion bounds from LEP, the tevatron and the LHC, Eur. Phys. J. C 74, 2693 (2014).

[88] F. Staub, Exploring new models in all detail with SARAH, Adv. High Energy Phys. 2015, 840780 (2015).

[89] A. Vicente, Computer tools in particle physics, arXiv: 1507.06349.

[90] W. Porod, SPheno, a program for calculating supersymmetric spectra, SUSY particle decays and SUSY particle production at $e+e-$ colliders, Comput. Phys. Commun. 153, 275 (2003).

[91] W. Porod and F. Staub, SPheno 3.1: Extensions including flavour, $C P$-phases and models beyond the MSSM, Comput. Phys. Commun. 183, 2458 (2012).
[92] W. Porod, F. Staub, and A. Vicente, A Flavor Kit for BSM models, Eur. Phys. J. C 74, 2992 (2014).

[93] J. Camargo-Molina, B. O’Leary, W. Porod, and F. Staub, VEVACIOUS: A tool for finding the global minima of one-loop effective potentials with many scalars, Eur. Phys. J. C 73, 2588 (2013).

[94] J. Camargo-Molina, B. Garbrecht, B. O’Leary, W. Porod, and F. Staub, Constraining the Natural MSSM through tunneling to color-breaking vacua at zero and non-zero temperature, Phys. Lett. B 737, 156 (2014).

[95] C. L. Wainwright, Cosmotransitions: Computing cosmological phase transition temperatures and bubble profiles with multiple fields, Comput. Phys. Commun. 183, 2006 (2012).

[96] J. L. Hintze and R. D. Nelson, Violin plots: A box plotdensity trace synergism, Am. Stat. 52, 181 (1998).

[97] P. Athron et al. (GAMBIT Collaboration), Global fits of GUT-scale SUSY models with GAMBIT, Eur. Phys. J. C 77, 824 (2017).

[98] S. Profumo, T. Stefaniak, and L. Stephenson Haskins, The not-so-well tempered neutralino, Phys. Rev. D 96, 055018 (2017).

[99] E. Bagnaschi et al., Likelihood analysis of the pMSSM11 in light of LHC 13-TeV data, Eur. Phys. J. C 78, 256 (2018).

[100] K. Kowalska and E. M. Sessolo, The discreet charm of higgsino dark matter-a pocket review, Adv. High Energy Phys. 2018, 6828560 (2018).

[101] K. Griest and D. Seckel, Three exceptions in the calculation of relic abundances, Phys. Rev. D 43, 3191 (1991).

[102] M. Benabderrahmane, Latest results from the XENON1T experiment, J. Phys. Conf. Ser. 1258, 012009 (2019).

[103] G. Aad et al. (ATLAS Collaboration), Search for electroweak production of charginos and sleptons decaying into final states with two leptons and missing transverse momentum in $\sqrt{s}=13 \mathrm{TeV} p p$ collisions using the ATLAS detector, Eur. Phys. J. C 80, 123 (2020).

[104] J. A. Evans and J. Shelton, Long-lived staus and displaced leptons at the LHC, J. High Energy Phys. 04 (2016) 056.

[105] S. Banerjee, G. Bélanger, A. Ghosh, and B. Mukhopadhyaya, Long-lived stau, sneutrino dark matter and right-slepton spectrum, J. High Energy Phys. 09 (2018) 143.

[106] S. Kraml, S. Kulkarni, U. Laa, A. Lessa, W. Magerl, D. Proschofsky-Spindler, and W. Waltenberger, sModels: A tool for interpreting simplified-model results from the LHC and its application to supersymmetry, Eur. Phys. J. C 74, 2868 (2014).

[107] F. Ambrogi et al., SModels v1.2: Long-lived particles, combination of signal regions, and other novelties, Comput. Phys. Commun. 251, 106848 (2020).

[108] M. Drees, H. Dreiner, D. Schmeier, J. Tattersall, and J. S. Kim, CheckMATE: Confronting your favourite new physics model with LHC data, Comput. Phys. Commun. 187, 227 (2015).

[109] D. Dercks, N. Desai, J. S. Kim, K. Rolbiecki, J. Tattersall, and T. Weber, CheckMATE 2: From the model to the limit, Comput. Phys. Commun. 221, 383 (2017). 
[110] J. S. Kim, D. Schmeier, J. Tattersall, and K. Rolbiecki, A framework to create customised LHC analyses within CheckMAte, Comput. Phys. Commun. 196, 535 (2015).

[111] W. Beenakker, R. Hopker, and M. Spira, PROSPINO: A program for the production of supersymmetric particles in next-to-leading order QCD, arXiv:hep-ph/9611232.

[112] J. Alwall, M. Herquet, F. Maltoni, O. Mattelaer, and T. Stelzer, MadGraph 5: Going beyond, J. High Energy Phys. 06 (2011) 128.

[113] E. Conte, B. Fuks, and G. Serret, MadAnalysis 5, A userfriendly framework for collider phenomenology, Comput. Phys. Commun. 184, 222 (2013).

[114] T. Sjöstrand, S. Ask, J. R. Christiansen, R. Corke, N. Desai, P. Ilten, S. Mrenna, S. Prestel, C. O. Rasmussen, and P.Z. Skands, An introduction to PYTHIA 8.2, Comput. Phys. Commun. 191, 159 (2015).

[115] J. de Favereau, C. Delaere, P. Demin, A. Giammanco, V. Lemaître, A. Mertens, and M. Selvaggi (DELPHES 3 Collaboration), DELPHES 3, A modular framework for fast simulation of a generic collider experiment, J. High Energy Phys. 02 (2014) 057.

[116] G. Aad et al. (ATLAS Collaboration), Search for charginoneutralino production with mass splittings near the electroweak scale in three-lepton final states in $\sqrt{s}=13 \mathrm{TeV} p p$ collisions with the ATLAS detector, Phys. Rev. D 101, 072001 (2020).

[117] A. Barr, C. Lester, and P. Stephens, m(T2): The truth behind the glamour, J. Phys. G 29, 2343 (2003).

[118] G. Aad et al. (ATLAS Collaboration), Searches for electroweak production of supersymmetric particles with compressed mass spectra in $\sqrt{s}=13 \mathrm{TeV} p p$ collisions with the ATLAS detector, Phys. Rev. D 101, 052005 (2020).
[119] G. Aad et al. (ATLAS Collaboration), Search for charginoneutralino pair production in final states with three leptons and missing transverse momentum in $\sqrt{s}=13 \mathrm{TeV} p p$ collisions with the ATLAS detector, arXiv:2106.01676.

[120] M. Aaboud et al. (ATLAS Collaboration), Search for electroweak production of supersymmetric states in scenarios with compressed mass spectra at $\sqrt{s}=13 \mathrm{TeV}$ with the ATLAS detector, Phys. Rev. D 97, 052010 (2018).

[121] G. Aad et al. (ATLAS Collaboration), Search for direct production of electroweakinos in final states with one lepton, missing transverse momentum and a Higgs boson decaying into two $b$-jets in $p p$ collisions at $\sqrt{s}=13 \mathrm{TeV}$ with the ATLAS detector, Eur. Phys. J. C 80, 691 (2020).

[122] D. R. Tovey, On measuring the masses of pair-produced semi-invisibly decaying particles at hadron colliders, J. High Energy Phys. 04 (2008) 034.

[123] G. Polesello and D. R. Tovey, Supersymmetric particle mass measurement with the boost-corrected contransverse mass, J. High Energy Phys. 03 (2010) 030.

[124] A. Sirunyan et al. (CMS Collaboration), Search for supersymmetry in final states with two oppositely charged same-flavor leptons and missing transverse momentum in proton-proton collisions at $\sqrt{s}=13 \mathrm{TeV}$, J. High Energy Phys. 04 (2021) 123.

[125] A.L. Read, Presentation of search results: The CL(s) technique, J. Phys. G 28, 2693 (2002).

[126] G. Schott (RooStats Team Collaboration), Roostats for Searches, in PHYSTAT 2011 (CERN, Geneva, 2011), pp. 199-208.

[127] L. Morel, Z. Yao, P. Clad, and S. Guellati-Khlifa, Determination of the fine-structure constant with an accuracy of 81 parts per trillion, Nature (London) 588, 61 (2020). 